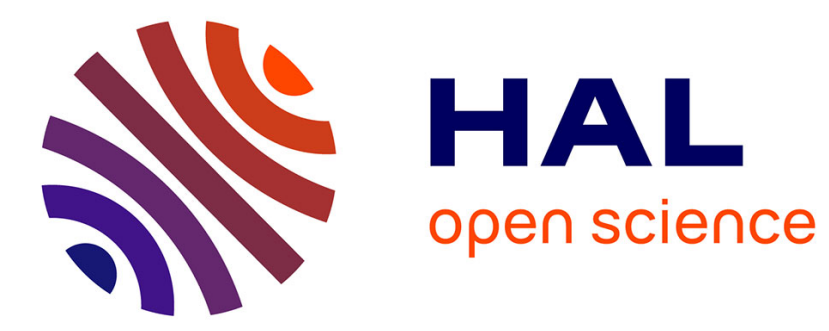

\title{
Coupled convection and internal gravity waves excited in water around its density maximum at $4^{\circ} \mathrm{C}$
}

Pierre Léard, Benjamin Favier, Pierre Le Gall, Michael Le Bars

\section{To cite this version:}

Pierre Léard, Benjamin Favier, Pierre Le Gall, Michael Le Bars. Coupled convection and internal gravity waves excited in water around its density maximum at $4^{\circ} \mathrm{C}$. Physical Review Fluids, 2020, 5, 10.1103/physrevfluids.5.024801 . hal-03011310

\section{HAL Id: hal-03011310 https://hal.science/hal-03011310}

Submitted on 18 Nov 2020

HAL is a multi-disciplinary open access archive for the deposit and dissemination of scientific research documents, whether they are published or not. The documents may come from teaching and research institutions in France or abroad, or from public or private research centers.
L'archive ouverte pluridisciplinaire HAL, est destinée au dépôt et à la diffusion de documents scientifiques de niveau recherche, publiés ou non, émanant des établissements d'enseignement et de recherche français ou étrangers, des laboratoires publics ou privés. 


\title{
Coupled convection and internal gravity waves excited in water around its density maximum at $4^{\circ} \mathrm{C}$
}

\author{
P. Léard ${ }^{\circ},{ }^{*}$ B. Favier, P. Le Gal, and M. Le Bars \\ Aix Marseille Univ, CNRS, Centrale Marseille, IRPHE, Marseille, France
}

(Received 3 October 2019; accepted 19 December 2019; published 5 February 2020)

\begin{abstract}
Coupled mixed convective and stratified systems are common in natural flows. To study experimentally the associated dynamics, we use a singular property of water: its nonlinear equation of state is characterized by a maximum density close to $4^{\circ} \mathrm{C}$. By heating the top of a tank at $35^{\circ} \mathrm{C}$ and cooling the bottom at $0{ }^{\circ} \mathrm{C}$, a two-layer configuration spontaneously appears. The convective motion in the bottom layer consists mostly of a large-scale circulation and rising cold plumes. This turbulent flow generates propagating internal gravity waves in the upper stably stratified layer. Particle image velocimetry measurements are performed and spectral characteristics of the convection and internal gravity waves are presented. An horizontal large-scale reversing flow in the stratified layer is observed which is viscously driven by a third, intermediate layer. This buffer layer is located between the convective and stratified layers and is thermally coupled with the convective one, hence sustaining a strong horizontal shear. Three-dimensional direct numerical simulations with geometry and physical parameters close to the experimental ones corroborate our experimental results.
\end{abstract}

DOI: 10.1103/PhysRevFluids.5.024801

\section{INTRODUCTION}

Numerous geophysical and astrophysical flows present a two-layer configuration, with a turbulent convective layer standing above or below a stably stratified one. Examples include planetary atmospheres, stars interiors, and possibly the outermost layer of the Earth's liquid core [1]. The dynamics of coupled stratified and convective layers are quite complex. Due to the convective motions, internal gravity waves (IGWs) are generated at the interface between the two layers, and propagate in the stratified one. IGWs transport energy and momentum [2,3] from where they are generated to where they are damped. Thanks to their transport properties and nonlinear interactions, IGWs are able to generate and sustain large-scale horizontal flows [2,4]. Examples of such large-scale flows driven by IGWs are the oscillations of equatorial zonal winds observed in some planets' atmospheres [5,6], including the Earth where it is called the quasibiennial oscillation (QBO) [7].

The IGW generation by turbulent dynamics has been studied in various experiments. The generation by a single buoyant plume was experimentally studied by Ansong and Sutherland [8]. The penetration of the plume within the stratified layer and the spectral characteristics of the generated IGWs were studied. They found that the peak frequency of the generated IGWs lies in a range close to $0.7 N$, where $N$ is the Brunt-Väisälä (or buoyancy) frequency, and that the radial wave number is set by the plume cap and not by the width of the plume at the interface.

Deardoff et al. [9] and later Michaelian et al. [10] studied the effect of penetrative convection in a stratified layer in a transient, Rayleigh-Bénard type experiment. Stratification was initially set

*leard@irphe.univ-mrs.fr 
up thermally from the top to the bottom of the tank. Then, the fluid was suddenly warmed up at the bottom, triggering Rayleigh-Bénard convection. IGWs were measured transiently [10] while the stratified (resp. convective) layer was decreasing (resp. increasing) in size. Eventually, there was no more stratified layer to sustain the propagation of IGWs.

Townsend [11] introduced an original setup to study the quasisteady generation of IGWs by Rayleigh-Bénard convection. Using the fact that water maximum density is around $4{ }^{\circ} \mathrm{C}$, a two-layer system is spontaneously generated by cooling the bottom of a tank at $0^{\circ} \mathrm{C}$ and heating its top above $4{ }^{\circ} \mathrm{C}$. The density gradient is unstable at temperatures below $4{ }^{\circ} \mathrm{C}$ and stable at temperatures above. This creates a self-organizing system, with a turbulent convective layer adjacent to a stratified layer. With dye visualization and temperature measurements, he observed IGWs propagating close to the interface between the two layers. The $4{ }^{\circ} \mathrm{C}$ convection was also studied experimentally by Le Gal [12] in a laminar flow, at low Rayleigh number. Convection displayed an hexagonal pattern and viscous entrainment of the fluid above the convective cells was observed. Perrard et al. [13] and Le Bars et al. [14] reinvestigated this setup in a quasi-two-dimensional tank using particle image velocimetry (PIV) and temperature measurements to obtain detailed data of IGWs generated by the convection. They observed a wide spectrum of waves generated at different frequencies. Favored frequencies were related to the differential attenuation length of the waves depending on frequency, in good agreement with linear theory. No large-scale flow in the stratified layer was observed in this two-dimensional (2D) geometry. Numerical simulations of the same configuration were performed by Lecoanet et al. [15]. They demonstrated that IGWs are mainly generated by the Reynolds stresses in the bulk of the convection below the interface, rather than by the displacement of the interface between the two layers. Numerical studies by Couston et al. [16-18] extended these results by considering a generic nonlinear equation of state (piecewise linear around an inversion temperature, with adjustable slopes), in both 2D and 3D horizontally periodic geometries. Various flow regimes and the energy transport by IGWs were quantitatively studied. Interestingly, long simulationsaccessible in 2D studies only-showed, for low Prandtl numbers $(\operatorname{Pr}<1)$, a large-scale horizontal flow with reversals in the stratified layer, similar to the QBO phenomenon introduced above [17].

Several experiments took interest in the generation and reversal of a large-scale horizontal mean flow in a stratified domain, driven by IGWs. The well-known experiment designed by Plumb and McEwan [19], later reproduced and extended by Semin et al. [20,21], is capable of driving a QBO from the mechanical forcing of a standing wave pattern (i.e., two waves with the same frequency and opposite phase speed) in a salty water stratification. With this system, Plumb and McEwan managed to observe a few oscillations of the driven large-scale flow before the stratification was destroyed by mixing. The experiment gave results in good agreement with the theory $[4,22,23]$, notably with reversals starting away from the forcing and propagating towards it. Semin et al. [20,21] improved the system by constantly injecting fluid to rebuild the stratification, while removing mixed fluid close to the forcing. This method allowed them to run the experiment longer and to study the nature of the bifurcation in the Plumb model, which can be either supercritical or subcritical, depending on the dominant dissipative process. In those experimental realizations of the QBO mechanism, the wave forcing remains monochromatic, as opposed to the natural mechanism where it is due to chaotic tropical storms [7]. The forcing is driven by interface displacements, as opposed to the observations of [15]. Besides, only the stratified layer is modeled. It thus remains a challenge to observe experimentally a large-scale, reversing flow from a turbulent source and a wide range of naturally excited IGWs.

In the present study, we extend the work of Townsend $[11,13,14]$ in a cylindrical, 3D geometry reminiscent of Plumb and McEwan's setup [19-21]. Our purpose is threefold: to characterize the generation of IGWs in such a self-organizing two-layer system, to quantify the coupling between the layers, and to investigate the possible generation of large-scale horizontal flows. Our experiments are complemented by direct numerical simulations of the same configuration. The experimental setup and numerical model are presented in Sec. II, results are analyzed in Sec. III, and conclusions and future works are discussed in Sec. IV. 


\section{METHODS}

\section{A. Experimental setup}

The setup consists of a cubic tank whose lateral boundaries are made of $2 \mathrm{~cm}$ thick acrylic walls. The bottom boundary is a chrome plated copper plate in which refrigerated fluid is forced to circulate. The top boundary is a commercial, transparent electric heater. The tank inner dimensions are $32 \times 32 \mathrm{~cm}^{2}$ for the horizontal section and $H=20 \mathrm{~cm}$ in height. Preliminary experiments were conducted in this cubic geometry. Eventually, a cylinder of outer diameter $D=29 \mathrm{~cm}$ and thickness $e=0.4 \mathrm{~cm}$ was added inside the cubic tank, to reproduce the axisymmetric geometry of [19-21], which seems prone to the development of large-scale flows. We are interested in the flow within the cylinder: the fluid in the gap between the cylinder and the cubic tank follows a similar dynamics and thus imposes to the working fluid a (close to) no-flux lateral boundary condition.

The temperature of the bottom boundary is controlled by water circulating in the copper plate. Water is cooled down by a thermal bath with a fixed temperature set at $-1.25^{\circ} \mathrm{C}$. Due to thermal losses in the pipes alimenting the copper plate, the bottom tank temperature is $0.2 \pm 0.05^{\circ} \mathrm{C}$. Plate thickness and circulation circuit were optimized so as to ensure a uniform temperature over the whole plate. At the top boundary, the heater is set at a temperature of $35^{\circ} \mathrm{C}$. Its temperature control is custom made: a PT100 probe measures the heater temperature in real time, driving through a feedback loop the input power injected in the heater. This is a very simple and inexpensive system to impose a temperature while having a transparent top boundary, allowing visualization and velocity measurements by PIV. Nonetheless, it is necessary to point out that the temperature over the heater area is not perfectly homogeneous. Temperature is maximal at the center, where $T \sim 38^{\circ} \mathrm{C}$, while the edges are indeed at the targeted $T=35 \pm 0.1{ }^{\circ} \mathrm{C}$. This inhomogeneity of the top temperature by $\delta T=3{ }^{\circ} \mathrm{C}$ induces slow convective motions below the heater, in a $\sim 2 \mathrm{~cm}$ high layer. By performing an experiment where the whole fluid is stably stratified with an overall temperature gradient similar to the one in the stratified layer studied here, but above the density reversal at $4{ }^{\circ} \mathrm{C}$ (i.e., bottom boundary set at $10^{\circ} \mathrm{C}$ and top boundary at $70^{\circ} \mathrm{C}$ ), we have checked that those top convective motions have no significant impact on the dynamics of the two-layer system. It is also important to say that, despite the thick acrylic wall and the intermediate fluid layer between the cylinder and the tank, the working region is not fully thermally insulated on the sides. Nevertheless, our fully stratified test experiment has shown no motion within the fluid driven by these lateral losses.

The equation of state of water is nonlinear with a maximum density close to $4{ }^{\circ} \mathrm{C}$ (International Equation of State of Seawater, 1980):

$$
\begin{aligned}
\rho(T)= & 999.842594+6.793952 .10^{-2} T-9.095290 .10^{-3} T^{2}+1.001685 .10^{-4} T^{3} \\
& -1.120083 .10^{-6} T^{4}+6.536332 .10^{-9} T^{5} .
\end{aligned}
$$

Thus, due to the imposed temperatures at top and bottom boundaries, the bottom part of the tank, between $0{ }^{\circ} \mathrm{C}$ and $4{ }^{\circ} \mathrm{C}$, is convectively unstable (see Fig. 1). Cold plumes detach from the bottom plate and rise in the tank due to buoyancy. Reciprocally, "hot" fluid sinks from the $4{ }^{\circ} \mathrm{C}$ interface due to gravity. While convective motion takes place in the lower layer, the upper part of the tank, between $4{ }^{\circ} \mathrm{C}$ and $35^{\circ} \mathrm{C}$, is stably stratified, with an assumed linear temperature profile at equilibrium. The temperature is indeed linear for an ideal case without thermal losses, assuming that the stratified layer has a bottom boundary at fixed temperature $4{ }^{\circ} \mathrm{C}$ and top boundary at $35^{\circ} \mathrm{C}$ (i.e., constant diffusive flux through the whole layer). However, the density profile is not linear, due to the nonlinear equation of state of water. Stratification is characterized by the Brunt-Väisälä frequency $N^{*}=\frac{1}{2 \pi} \sqrt{-\frac{g}{\rho_{0}} \frac{\partial \rho}{\partial z}}$. Because of the nonlinear equation of state, $N^{*}$ is not constant with depth, as shown in Fig. 1. For simplicity, we also use below the global buoyancy frequency defined as $N=\frac{1}{2 \pi} \sqrt{-\frac{g}{\rho_{0}} \frac{\Delta \rho}{\Delta z}}$, where $\Delta \rho$ is the global density contrast within the stratified layer of depth $\Delta z$.

Before starting the experiment, the bath and the heater are allowed to reach their assigned temperatures. Then, the upper half of the tank is filled with water stratified in temperature from $4{ }^{\circ} \mathrm{C}$ to $35^{\circ} \mathrm{C}$, using the double bucket technique [24]. The bottom half is filled with water with a 

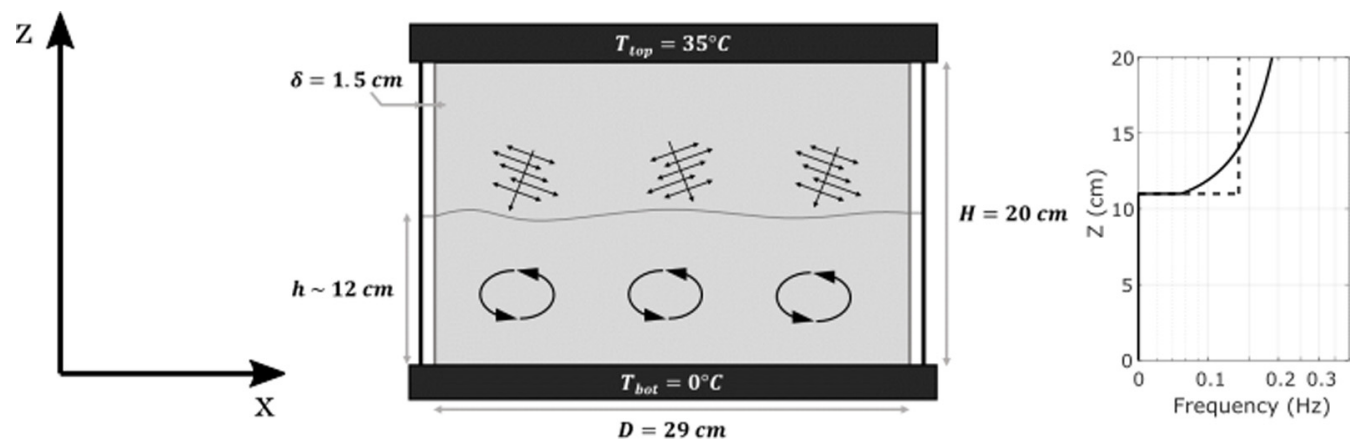

FIG. 1. 2D sketch of the tank. A cylinder (light grey shaded area) is placed in a larger cubic tank. The system is cooled down at the bottom at $0{ }^{\circ} \mathrm{C}$ and heated up at the top at $35^{\circ} \mathrm{C}$. The bottom half is convective with an almost constant density, apart from the bottom boundary layer. The upper half is stably stratified and waves are generated due to the fluid motions in the convective layer. The graph on the right shows the theoretical profile for the buoyancy frequency $N^{*}$. It is computed considering a linear temperature profile and the equation of state of water (1). The dashed line is the global buoyancy frequency $N$ calculated on the stratified layer. The various length scales are the cylinder diameter $D$, the vertical extent of the tank $H$ and the vertical extent of the convective layer $h . \delta$ is the minimal width between the outer square tank and the inner cylinder. The $x, y$ and $z$-velocity components are noted $u, v$ and $w$ respectively.

temperature close to $4{ }^{\circ} \mathrm{C}$. This filling process is used to avoid tremendously long transient before reaching steady state by thermal diffusion. Typically, we fill the tank at the end of a day and start the measurements the next day in order to let the system reach its equilibrium state overnight. Each experiment then lasts four days, with no change in the location of the interface. Note that this steady interface position is the result of the heat budget equilibrium between the convective heat flux extracted from the bottom plate, the diffusive heat flux through the stratified layer, and the lateral heat losses.

To perform PIV measurements, particles are chosen as small as possible and with a density as close as possible to water density in order to avoid their sedimentation in the stratified layer over the long duration of the experiment. We use fluorescent orange polyethylene microspheres whose size ranges from 10 to $20 \mu \mathrm{m}$ and density is $1.00 \pm 0.01 \mathrm{~g} / \mathrm{cm}^{3}$. The fluorescent property allows us, with a high pass filter on the camera, to remove any laser reflection, significantly enhancing the images quality. The tank is illuminated with a green laser of $532 \mathrm{~nm}$. Power is set at $1 \mathrm{~W}$. We perform side view PIV to measure convection and IGW spectral characteristics, and top view PIV to observe the large-scale flow and its fluctuations over time. The camera used for the side view PIV is a HiSense Zyla with $2560 \times 2160$ pixels recorded on 12 bits. The acquisition rate is $2 \mathrm{~Hz}$ with $100 \mathrm{~ms}$ exposure time. Typical acquisition time for spectral characteristics is $50 \mathrm{~min}$. For the top view, we use a Point grey camera with $1920 \times 1080$ pixels on 8 bits. Exposure time is $1 \mathrm{~s}$, acquisition rate $0.1 \mathrm{~Hz}$ and acquisition time 8 hours. Captured movies are processed either by the DantecDynamics software DYNAMICSTUDIO for the side view or by DPIVSOFT [25] for the top view. Both are resolved into $32 \times 32$ pixels boxes with $50 \%$ overlapping.

Side view PIVs are performed in the middle of the tank at $y=16 \mathrm{~cm}$ in a laser sheet crossing the cylinder along its diameter. This is the case for all figures shown in the $(x, z)$ plane and thus not mentioned in the results section. The vertical fields (see an example in Fig. 2) do not show the whole $(x, z)$ plane [where the origin $(0,0)$ is located in the bottom left corner of the cubic tank]: it was indeed chosen to zoom in, in order to have the best resolution for the very weak motions in the stratified layer. The interface between the layers is localized between $11 \leqslant z \leqslant 12 \mathrm{~cm}$. The typical Rayleigh number for the convection based on this depth is $\mathrm{Ra}=7 \times 10^{6}$, and the global Brunt-Väisälä frequency is $N=1.35 \times 10^{-1} \mathrm{~Hz}$. 

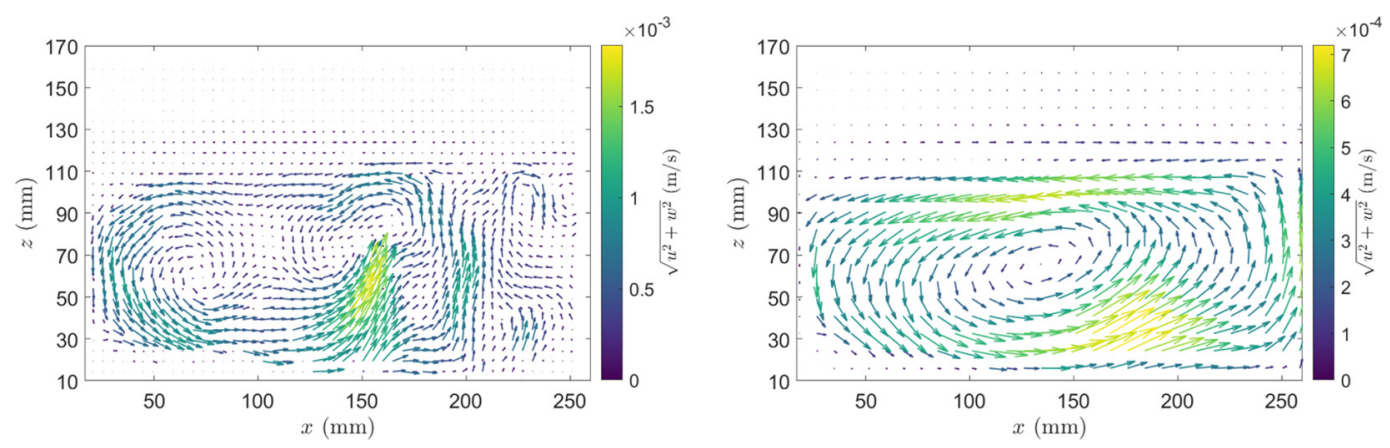

FIG. 2. Left: Instantaneous velocity field. An ascending plume is visible at $x=150 \mathrm{~mm}$, and transported by the large-scale circulation. Right: Large-scale circulation in the convective layer obtained by time-averaging velocities over a 50 minute signal. The large-scale circulation is a counterclockwise cell. No inversion of the circulation has been seen in our experiment. The velocities under $z=10 \mathrm{~mm}$ are noisy and thus not shown here. Maximum instantaneous velocities are 2.5 times bigger than the maximum averaged velocities. The left edge of the cylinder is located at $x=19 \mathrm{~mm}$ and the center of the cylinder is at $x=160 \mathrm{~mm}$. Approximately $40 \mathrm{~mm}$ of the right side of the cylinder is not captured with our PIV measurments.

To observe the large-scale flow, horizontal views at different heights are performed. A linear translation axis platform from Igus, driven by homemade software, is used to automate the process. With two mirrors, one fixed at the exit of the laser head and the other fixed on the translating platform, it is possible to sweep along one direction with the laser sheet. We typically make measurements during $15 \mathrm{~s}$ in each of 11 successive planes separated by $0.5 \mathrm{~cm}$. The full scan duration is about $3 \mathrm{~min}$, and is repeated during at least 8 hours.

The cylindrical geometry described here differs from the cylindrical shell geometry in [19-21]. We first tried to work in that annular geometry by adding a second, smaller cylinder in our cubic tank. Three different gap sizes were tested but none showed any interesting dynamics. Indeed, the convection was too confined in the shell to provide an efficient chaotic excitation, and IGWs did not propagate well within the stratification, because they were attenuated quite fast by wall friction. During these tests, we observed the most interesting dynamics within the innermost cylinder, so we decided to use that geometry. The point of the cylindrical shell geometry is that it is a close analog to the equatorial band of the stratosphere where QBO takes place. By working in a cylinder, the geometry analogy is lost but the physics of the problem remains the same, still a priori allowing for large-scale, reversing, axisymmetric horizontal flows.

\section{B. Numerical model}

To complement the experiments, we also performed direct numerical simulations (DNS) of the same configuration. We solve the Navier-Stokes equations using a non-Oberbeck Boussinesq model. The density variations are considered small compared to the reference density $\frac{\Delta \rho}{\rho_{o}} \ll 1$. Therefore, density fluctuations only appear in the buoyancy force. However, temperature variations affect the value of the thermal expansion coefficient to account for the nonlinear equation of state of water. Variations of the thermal diffusivity $\kappa$ and kinematic viscosity $v$ are neglected. Governing equations are given in dimensionless form by

$$
\begin{gathered}
\frac{\partial \boldsymbol{u}}{\partial t}+\boldsymbol{u} \cdot \nabla \boldsymbol{u}=-\nabla P+\operatorname{Pr} \nabla^{2} \boldsymbol{u}+\operatorname{Pr} \operatorname{Ra} \theta^{2} \boldsymbol{e}_{z} \\
\frac{\partial \theta}{\partial t}+\boldsymbol{u} \cdot \nabla \theta=\nabla^{2} \theta \\
\nabla \cdot \boldsymbol{u}=0
\end{gathered}
$$


where we used the depth $H$ of the container as a unit of length, the thermal diffusive timescale $H^{2} / \kappa$ as a unit of time, and the difference between the bottom and the inversion temperature $T_{0}-T_{i}$ as a temperature scale. These equations are characterized by the Prandtl number $\operatorname{Pr}=$ $\nu / \kappa$, the global Rayleigh number $\mathrm{Ra}=\alpha g\left(T_{0}-T_{i}\right)^{2} H^{3} /(\nu \kappa)$, and the imposed dimensionless top temperature $\theta_{\text {top }}=\left(T_{\text {top }}-T_{i}\right) /\left(T_{0}-T_{i}\right)$. Note that the quadratic temperature term in the momentum equation is a direct consequence of the nonlinear equation of state of water given by Eq. (1), which is approximated in our model by the quadratic equation $\rho(T) \approx \rho_{0}\left(1-\alpha\left(T-T_{i}\right)^{2}\right)$. The coefficient $\alpha$ is not the usual thermal expansion coefficient but has a unit of $\left({ }^{\circ} \mathrm{C}\right)^{-2}$ and is given by $\alpha \approx 8.1 \times 10^{-6}\left({ }^{\circ} \mathrm{C}\right)^{-2}$ (see also [15]).

We consider a cylindrical fluid cavity of diameter $D=3 H / 2$ as in the experiments. Both horizontal plates are assumed to be no-slip and with fixed temperature. The side wall is assumed to be no-slip and perfectly insulating. This is of course not the case in the experiment, for which lateral heat losses are inevitable and top temperature is not exactly constant, but the objective is to check whether the conclusions drawn from the experimental results are robust and do not depend on these effects. Since the experiment runs with water, we use $\operatorname{Pr}=7$. The Rayleigh number of the experiment is $\mathrm{Ra}=7 \times 10^{7}$ while its dimensionless top temperature is $\theta_{\text {top }}=-7.75$. If we were to run the simulation with these parameters, the interface will be located very close to the top boundary. It is not the case in the experiment because of the lateral heat losses, which tend to reduce the effective Rayleigh number. For that reason, and instead of taking into account these losses as in [15], we kept the insulating lateral boundaries and use the slightly adjusted parameters $\mathrm{Ra}=10^{7}$ and $\theta_{\text {top }}=-11$ instead, which leads to an interface located approximately at $z \approx 120 \mathrm{~mm}$, as in the experiment. The Rayleigh number could not be lowered under $10^{7}$ in order to keep the convective flow turbulent enough, thus we had to increase the top temperature to have the interface located at $z \approx 120 \mathrm{~mm}$.

We perform DNS of Eqs. (2)-(4) using the spectral element solver NEK5000 [26]. The global geometry is decomposed into hexahedral elements, with vertical refinement close to the horizontal boundaries and around the mid-plane where the inversion isotherm is expected to be located. Velocity, buoyancy, and pressure variables are represented as tensor product Lagrange polynomials of order $N$ and $N-2$ based on Gauss or Gauss-Lobatto quadrature points. The total number of grid points is given by $\mathcal{E} N^{3}$, where $\mathcal{E}$ is the number of elements. For all the results discussed in this paper, the number of elements is $\mathcal{E}=8960$ and we use a polynomial order of $N=11$. Numerical convergence was checked by increasing the polynomial order $N$. Time integration is performed with a third-order mixed implicit-explicit scheme. The simulations are initialized with a small amplitude buoyancy perturbation and a temperature profile varying linearly between the top and bottom boundaries. Simulations are run until a quasisteady state is reached, after which data are accumulated to compute statistics.

\section{RESULTS}

\section{A. Experiments}

\section{Convection}

The PIV side view is used to quantify horizontal and vertical velocities in the convection zone. Examples of vertical velocities measured at one point in a given location are shown in Fig. 3, for both ascending cold and descending hot structures. Measurements are consistent with the numerical simulations $[15,16]$ showing intense, localized, cold rising plumes and more diffusive descending plumes. Moreover, these structures are advected by a large-scale circulation encompassing the whole convective layer, as shown in Fig. 2.

Spectral analysis is performed to extract power spectral density (PSD) from the velocity signals. Figure 4 shows the PSD of the convection vertical velocity $w$. For the two panels, the spectrum is flat with a lot of energy for low frequencies, then the energy drops above some cutoff frequency. The left panel of Fig. 4 shows the vertical velocity PSD at a single point close to the top of the 

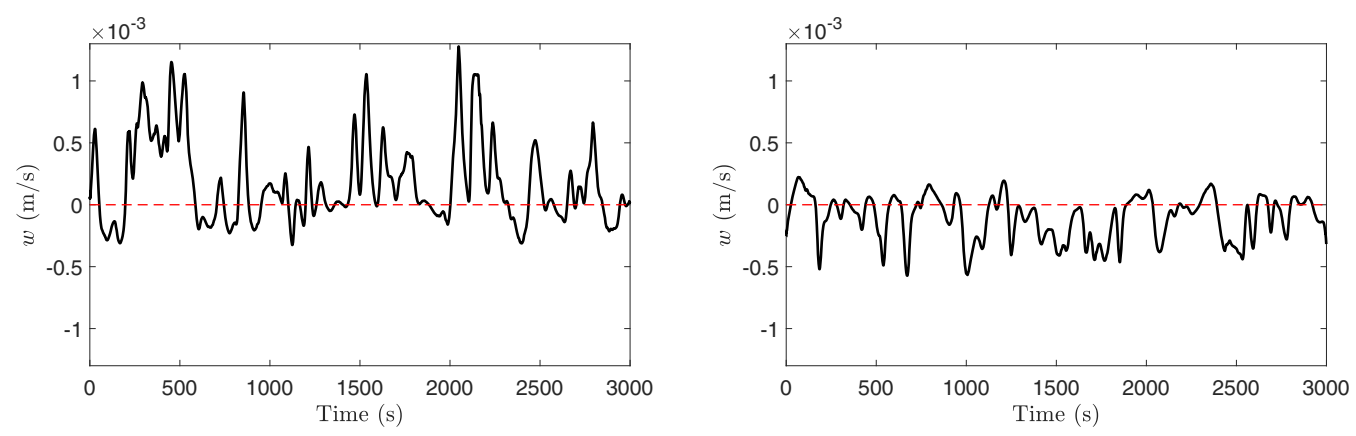

FIG. 3. Time evolution of the vertical velocity $w$ within (left) upward plumes at $x=200 \mathrm{~mm}, z=45 \mathrm{~mm}$ and (right) downward structures at $x=100 \mathrm{~mm}, z=95 \mathrm{~mm}$.

convective layer. A small peak can be seen close to $f=10^{-2} \mathrm{~Hz}$. This is the quasiperiodic signal of the plumes dropping from the top thermal boundary layer. The theoretical characteristic time of convection can be computed from [27]:

$$
\tau=\frac{h^{2}}{\pi \kappa}\left(\frac{\Delta T}{\Delta T_{\text {local }}} \times \frac{\mathrm{Ra}_{c}}{\mathrm{Ra}}\right)^{2 / 3}
$$

with $h$ the height of the convective layer, $\kappa$ the thermal diffusivity, $\Delta T$ the temperature difference between the top and bottom of the convective domain, $\Delta T_{\text {local }}$ the temperature difference between the top and bottom of the thermal boundary layer, and $\mathrm{Ra}_{c}$ the critical Rayleigh number. The critical Rayleigh number in the presence of free and solid interfaces and for fixed temperature is $\mathrm{Ra}_{c}=$ 1100.65. For our experiment, the characteristic time is $\tau=96 \mathrm{~s}$, thus characteristic frequency is $1 / \tau \sim 10^{-2} \mathrm{~Hz}$, which is close to the observed peak in the left panel of Fig. 4. At frequencies lower than this characteristic frequency, the spectrum is flat. This is explained by the combined effect of the randomness of the plumes (see Fig. 3) and of the slow fluctuations of the large-scale circulation. The right panel of Fig. 4 shows the PSD of vertical velocities, averaged over the whole convective cell in the $(x, z)$ plane. It shows a similar trend, with a lower cutoff frequency compared to the left panel spectrum. Actually, the plumes' signal is more localized and less intense on average than the large-scale circulation signal, which hence dominates the space-averaged PSD.

The probability density function (PDF) of the vertical velocities in the whole convective layer $P(w)$ is computed and shown in Fig 5. It is normalized such that $\int P(w) d w=1$. The PDF describes
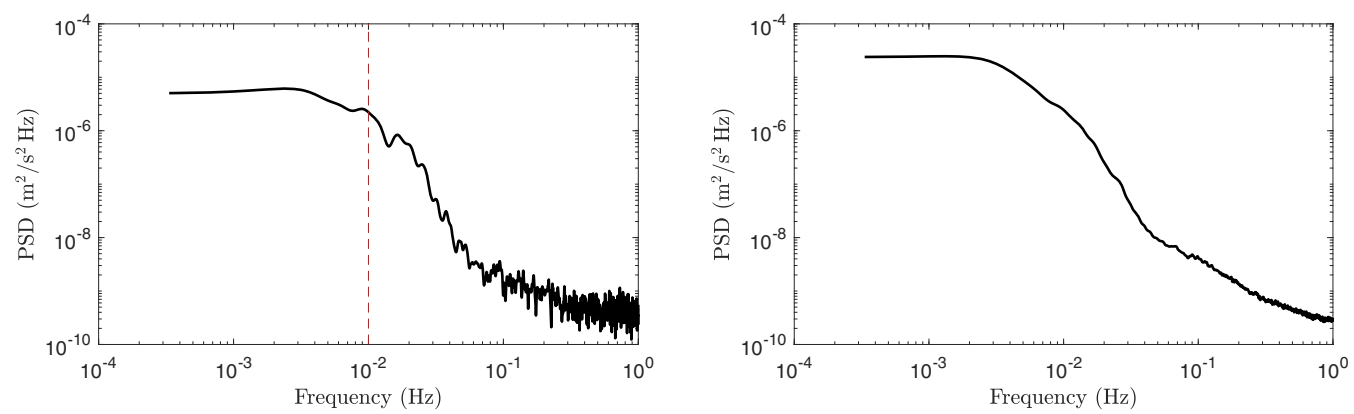

FIG. 4. PSD for the vertical velocity fluctuations. Left: PSD computed at a single point $x=100 \mathrm{~mm}$, $z=95 \mathrm{~mm}$ (signal shown in Fig. 3 right). The plume forcing frequency can be seen around $f=10^{-2} \mathrm{~Hz}$ (red dashed line). Right: PSD spatially averaged over the whole convective cell in the measured $(x, z)$ plane (all points above $z=10 \mathrm{~mm}$ and below $z=110 \mathrm{~mm}$ ). 


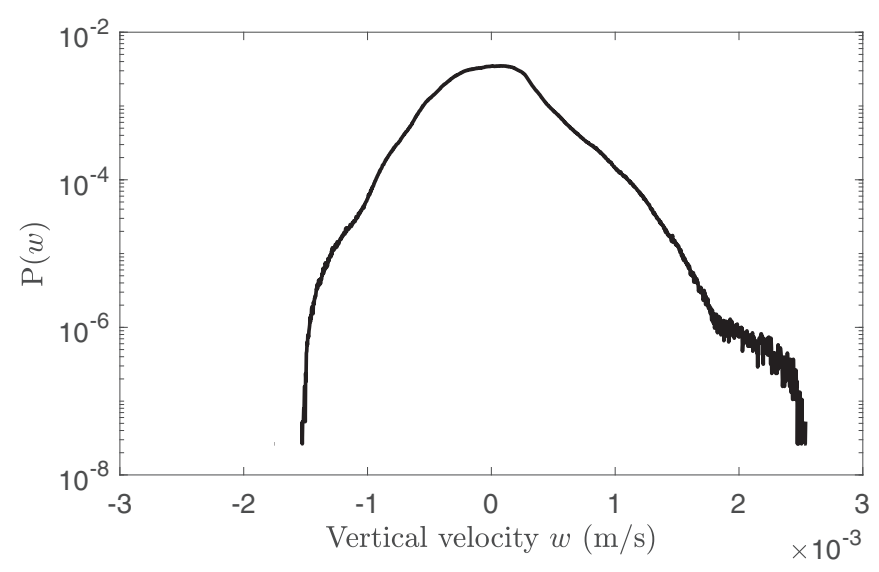

FIG. 5. Probability density function of the vertical velocities in the convective layer. All PIV points under $z=110 \mathrm{~mm}$ have been used to compute the PDF.

important features of the convection: it is skewed towards positive values, with positive velocities reaching higher magnitude than negative velocities; i.e., the ascending plumes are stronger than the descending structures. However, the central part of the PDF is close to a Gaussian profile. The distribution obtained here is in good agreement with the probability density function computed in an idealized 2D numerical model by Couston et al. [16]. Note that this asymmetry is specific to our model, for which the usual upside-down symmetry in Boussinesq Rayleigh-Bénard convection is broken.

\section{Buffer layer}

An intermediate layer (we name it the buffer layer in the following) is present between the convective layer and the stratified layer. It was first reported in the quasi-2D $4{ }^{\circ} \mathrm{C}$ convection experiment by Perrard et al. [13]. Their temperature measurements showed that the buffer layer corresponds to the area where the temperature goes from $4{ }^{\circ} \mathrm{C}$ to $8{ }^{\circ} \mathrm{C}$. This actually corresponds to the overshooting region for rising cold plumes (note that this type of convection is called "penetrative convection" because of this effect). Indeed, since the density of water is close to quadratic around $4{ }^{\circ} \mathrm{C}$, densities at, e.g., $0^{\circ} \mathrm{C}$ and $8{ }^{\circ} \mathrm{C}$ are the same, and the $8^{\circ} \mathrm{C}$ isotherm is the theoretical maximum height reachable by an ascending cold plume at $0^{\circ} \mathrm{C}$ in the absence of thermal diffusion. Simultaneously, the overall density profile between $4{ }^{\circ} \mathrm{C}$ and $8{ }^{\circ} \mathrm{C}$ is stable, as in the stratified layer above $8^{\circ} \mathrm{C}$. The buffer layer is thus a very specific location supporting simultaneously convective overshooting motions and IGWs, as observed with PIV measurements [13].

To complete the description of this buffer layer now using velocity measurements, we plot in Fig. 6 the spatiotemporal graph of the horizontal average of the horizontal velocity $u$. The graph exhibits a strong shear around $z=120 \mathrm{~mm}$. Since the fluid is going in opposite directions above and below $z=120 \mathrm{~mm}$ with a sharp interface, viscous entrainment by the convective layer is excluded. A special kind of thermal coupling might explain the observed dynamics, as sketched in Fig. 7. Indeed, when a cold ascending plume from the convection zone reaches the interface and overshoots in the buffer region, its associated velocity perturbation dissipates more rapidly than its temperature perturbation. Due to gravity, the distorted part of the buffer region tends to sink back to its initial state (pictured by the green arrows), while the fluid above the buffer layer moves towards the impact point of the plume to take the place of the falling water (pictured by the red arrows). The buffer layer then needs some compensating fluid from the convective layer. This mechanism works when the velocity perturbation of the plume at the interface dissipates more rapidly than the thermal 


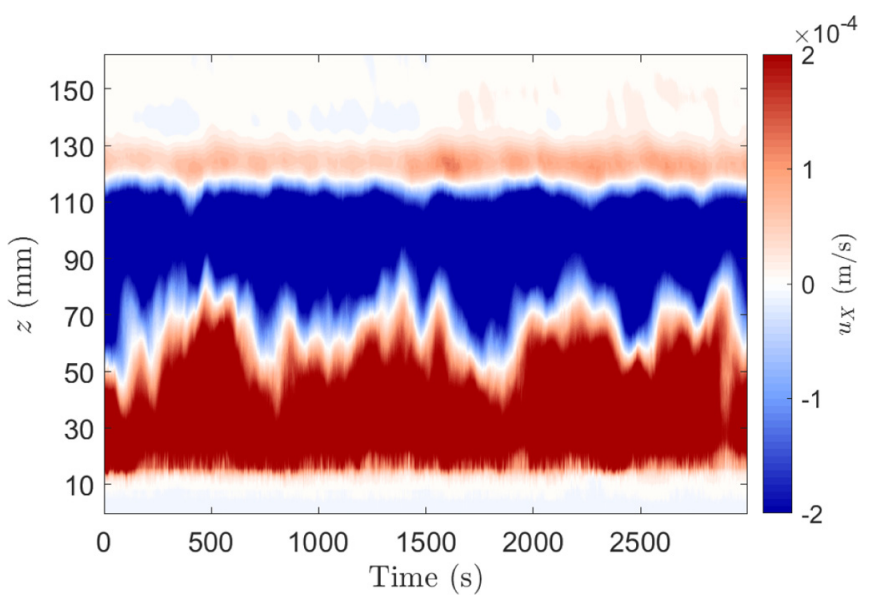

FIG. 6. Time evolution of the horizontal average of the horizontal velocity, noted $u_{X}$. Red (resp. blue) regions correspond to mean flow going towards the right (resp. left).

perturbation, hence for a Prandtl number $\operatorname{Pr} \geqslant 1$. One might expect the shearing zone to decrease in size and amplitude when thermal diffusion increases (i.e., when the Prandtl number decreases), since the overshooting rising cold plume will then equilibrate thermally during its ascent more rapidly. This may explain why no interfacial shear was reported in the systematic numerical study of $[16,17]$, where $\operatorname{Pr} \leqslant 1$. Global temperature field measurements (using, e.g., temperature dependent, laser induced fluorescence) are now required to confirm or infirm the proposed model, but those are beyond the scope of the present paper. Note that, by extension, we call "buffer layer" in the following the region including the $T=4{ }^{\circ} \mathrm{C}$ to $T=8{ }^{\circ} \mathrm{C}$ overshooting region and the shear region. In the experiment, the shear region extends from $z \approx 120 \mathrm{~mm}$ to $z \approx 135 \mathrm{~mm}$.

\section{Internal gravity waves}

The convective motions induce a Reynolds stress at and below the interface, which generates IGWs propagating in the stratified area [15]. An example is shown in Fig. 8. The vector field has been frequency filtered in the band $[0.02,0.03] \mathrm{Hz}$ to isolate a single propagating wave train. We can measure an angle close to $\theta \simeq 75^{\circ}$ between contant phase lines and the vertical. This observation is in good agreement with the inviscid dispersion relation $\omega= \pm N \cos (\theta)$, which relates the frequency and the propagation angle of IGWs. Indeed, at $z=120 \mathrm{~mm}, N \sim 0.1 \mathrm{~Hz}$, thus $\theta=\cos ^{-1}\left(\frac{\omega}{N}\right)=$ $78.5^{\circ}$. The motion within the stratified area is a superimposition of many such IGWs oscillating at different frequencies.
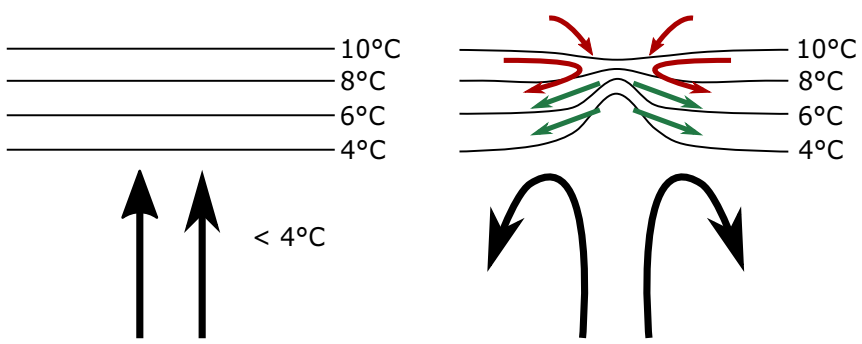

FIG. 7. Sketch of the thermal coupling between the convective and buffer layers. On the left, a cold plume moves upwards towards the interface between the two layers. On the right, isotherms are deflected due to the impact. 

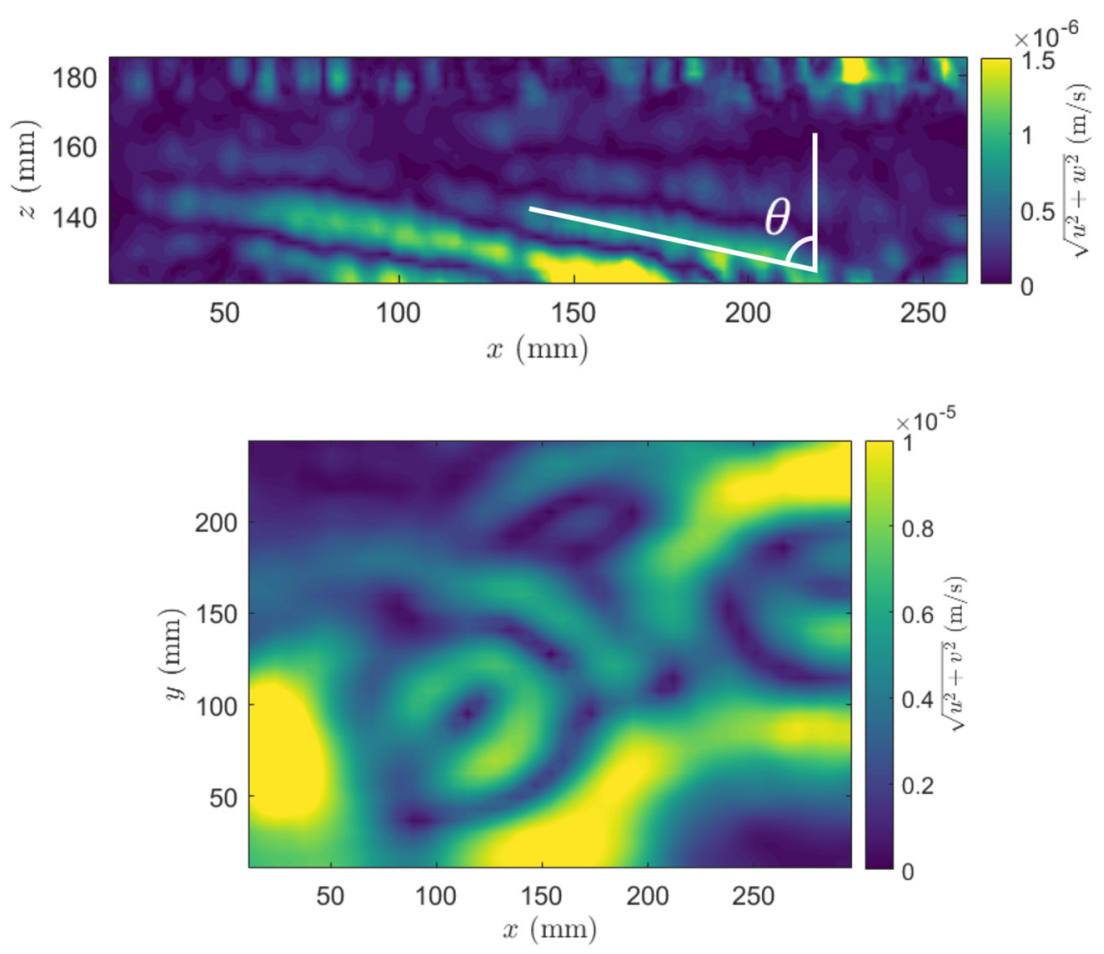

FIG. 8. Velocity fields showing IGWs propagating. Top: Velocities in $(x, z)$ plane. The signal is frequency filtered to enhance the visualization of oscillatory motions: only frequencies between 0.02 and $0.03 \mathrm{~Hz}$ are shown, propagating at an angle of roughly $75^{\circ}$ with the vertical. The angle of propagation is the angle between the constant phase line and the vertical. Bottom: Velocities in $(x, y)$ plane located at $z \approx 125 \mathrm{~mm}$. In the $(x, y)$ plane, IGWs take the form of oscillating rings. Note that this figure is from a previous experiment without any internal cylinder and is therefore only displayed here as an illustration of the IGWs seen from above.

To further investigate the waves' signal, wave spectra are plotted in Fig. 9, showing the power spectral density of oscillatory motions within the stratified layer at every height, averaged horizontally for each height. The grey line is the theoretically computed buoyancy frequency profile. Figure 9 shows that energy is present in a wide frequency band, from the lowest measured frequencies to the buoyancy frequency $N$. Low frequency motions $f<4 \times 10^{-3} \mathrm{~Hz}$ are very intense and propagate high in the stratified layer. Motions with frequency ranging from $4 \times 10^{-3} \mathrm{~Hz}$ to $\mathrm{N}$ are less intense, but still propagate into the stratified layer. Motions propagating at frequencies higher than the buoyancy frequency $N$ are greatly attenuated after a centimeter, as IGWs of frequency larger than $N$ are evanescent. The weak signal at low frequencies above $z=180 \mathrm{~mm}$ comes from the convective motions due to the non-homogeneous heating at the top. These motions are confined at the very top of the experimental container.

The right panel of Fig. 9 shows two vertical profiles of the PSD rescaled by the PSD at the top of the convective layer $(z=120 \mathrm{~mm})$, taken at two different frequencies. The energy decrease is quite similar between $z=120 \mathrm{~mm}$ and $z=140 \mathrm{~mm}$ for both frequencies. However, for $z>140 \mathrm{~mm}$, the energy for the higher frequency decreases slower than the energy for the lower frequency. This dependence of the attenuation length regarding the frequency of the signal is a characteristic of IGWs. Indeed, the dispersion relation of IGWs relates the frequency and the wave vector direction. Moreover, energy propagates perpendicularly to the wave vector for IGWs (i.e., group and phase velocities are perpendicular). The closer to $N$ the wave frequency $\omega$ is, the more horizontal the phase propagates, hence the more vertical energy propagates. High frequency waves are thus capable of 

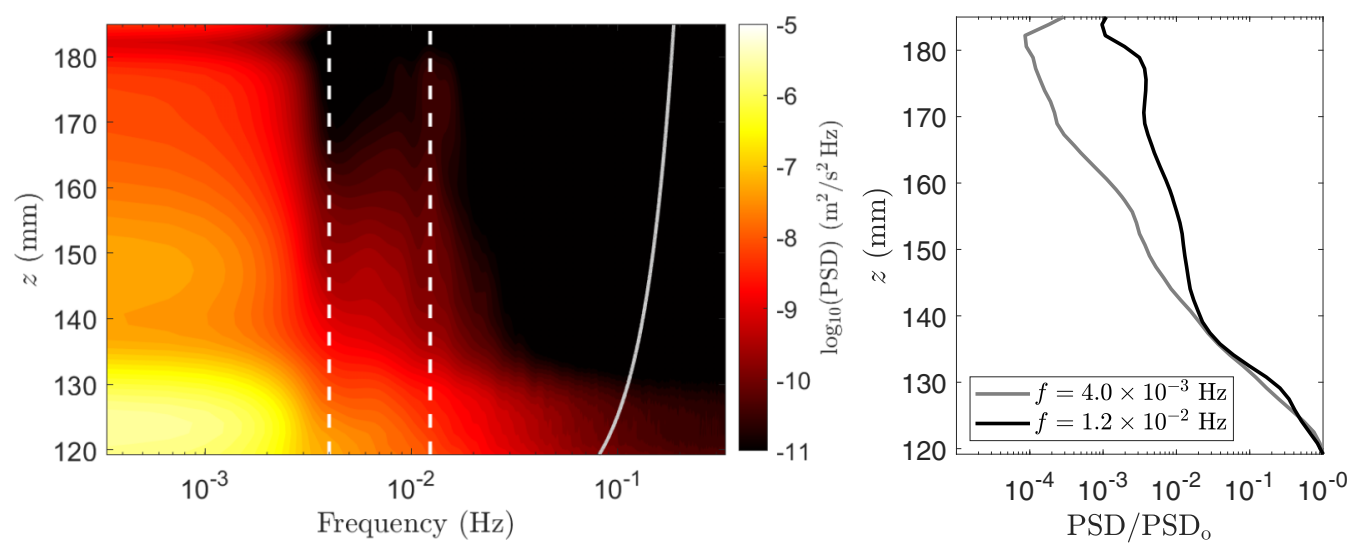

FIG. 9. Left: Power spectral density of the absolute velocity $\sqrt{u^{2}+w^{2}}$ above the convective layer. The grey curve shows the theoretical buoyancy frequency profile, assuming a linear profile for the temperature, from $4{ }^{\circ} \mathrm{C}$ to $35^{\circ} \mathrm{C}$. Right: Two selected profiles (taken at frequencies shown by dashed lines on the left graph) of the rescaled PSDs by the PSD at the top of the convective layer, i.e., $z=120 \mathrm{~mm}$ (denoted PSD ). The PIV measurements are performed for 50 minutes and results (using the pwelch MATLAB function) are horizontally averaged at each height to obtain the averaged power spectral density.

transporting energy to high altitudes before being damped. In contrast, waves with low frequency compared to $N$ propagate energy almost horizontally, and are thus attenuated before reaching high altitudes. At frequencies $f<4 \times 10^{-3} \mathrm{~Hz}$, a lot of energy is seen and the attenuation length does not depend on the frequency. There is no reason why IGWs should disappear below a certain frequency, but we would expect to see the attenuation length keep decreasing with decreasing frequency. We thus deduce that IGWs at frequencies $f \leqslant 4 \times 10^{-3} \mathrm{~Hz}$ are hidden in the energy spectrum by some very energetic large-scale slowly varying flow, which we will describe below.

More than one order of magnitude separates the buoyancy frequency and the fastest large-scale flow fluctuations. The large-scale flow penetrates deep into the stratified layer. It globally decreases in amplitude with height, but with some local increases at $z \sim 125 \mathrm{~mm}$ (i.e., close to the interface between convective and buffer layers) and $z \sim 145 \mathrm{~mm}$. The IGWs' signal can be seen between $f=4 \times 10^{-3} \mathrm{~Hz}$ and the buoyancy frequency. A peak that reaches the top of the stratified layer is seen around $f=1.2 \times 10^{-2} \mathrm{~Hz}$, i.e., the same frequency as the convective forcing discussed in Sec. III A 1. It corresponds to the strong excitation provided by the cold rising and hot sinking turbulent plumes. However, the top panel of Fig. 9 also shows a sudden drop of the energy at frequencies $f>1.2 \times 10^{-2} \mathrm{~Hz}$. Indeed, wave attenuation is strong at these frequencies, even if they are close to (but below) the buoyancy frequency $N$. Actually, energy dissipation also depends on the norm of the wave vector squared. There is no reason that all excited waves have the same wave vector norm; one could even expect that fastest waves are excited by fastest, hence smallest convective patterns, and are thus also at smallest scale: they then dissipate more rapidly.

\section{Large-scale flow in the stratified layer}

Figure 9 shows an important amount of energy at low frequencies which has been interpreted as the signature of a large-scale slowly varying flow in the stratified layer. We will now investigate the nature of these fluctuations to see if they relate to reversals similar to the QBO.

Figure 10 shows horizontal vector fields at the same depth at different times. In Fig. 10(a), the flow goes counterclockwise inside the cylinder. Figure 10(b) shows that two contrarotating vortices with smaller amplitude typical velocities have appeared. Figure 10(c) shows a mostly clockwise rotating flow, where one of the preceding eddy pairs has nearly disappeared. The large-scale flow thus evolves drastically over time. A criterion is computed to extract a typical mean velocity from 


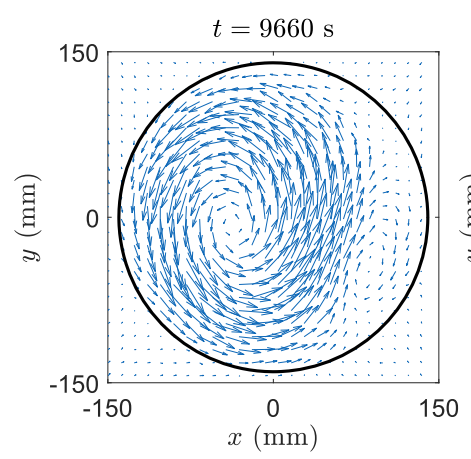

(a)

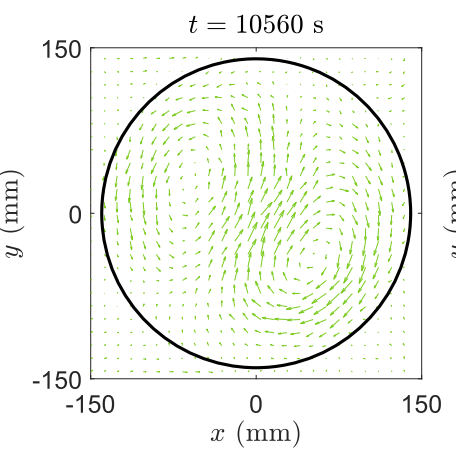

(b)

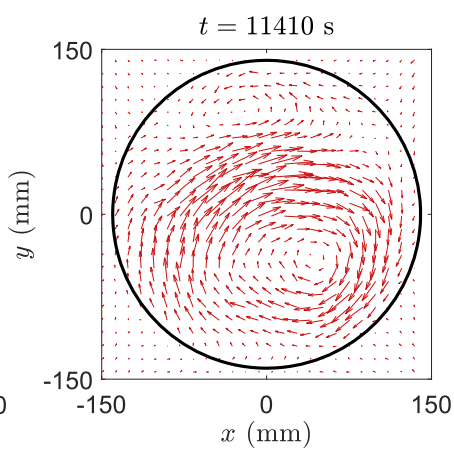

(c)

FIG. 10. Horizontal velocity vector fields in the stratified layer at different times. The laser sheet is located at $z=150 \mathrm{~mm}$. The large-scale flow reverses from (a) to (c). Time between (a) and (c) is approximately half an hour. Maximum velocities are $0.1 \mathrm{~mm} / \mathrm{s}$.

those fields that accounts for the "direction" of the large-scale flow: as illustrated in Fig. 11, we compute a mean azimuthal velocity, taken along a ring centered in the cylinder. Other criteria to extract a representative value for the large-scale flow direction have also been tested, including the mean vorticity over the cylinder area, the average of the azimuthal velocity over several rings with different radii, and the azimuthal velocity averaged over thick rings. They all give similar results for the large-scale flow measurement.

In order to investigate the vertical phase propagation of the reversals, and thus to compare the reversal dynamics observed to a QBO-like phenomenon, the setup has been equipped with a linear translating platform that allows us to perform horizontal laser sheet sweeping along the vertical. Horizontal velocities are measured in an horizontal plane, every $5 \mathrm{~mm}$ from the top of the convective layer $z=110 \mathrm{~mm}$ to the middle of the stratified layer $z=160 \mathrm{~mm}$. Any trace of downward phase propagation of the reversals, as observed on the QBO on Earth [7] and on the historical Plumb experiment $[4,21]$, would be a significant evidence for a QBO-like phenomenon in the experiment. Indeed, the phase propagation of the reversals due to IGW nonlinear interactions is theorized as follows: an IGW propagating in a stratified layer with an horizontal phase velocity in the same direction as the existing base flow propagates upward until reaching a critical height $z_{c}$, where it

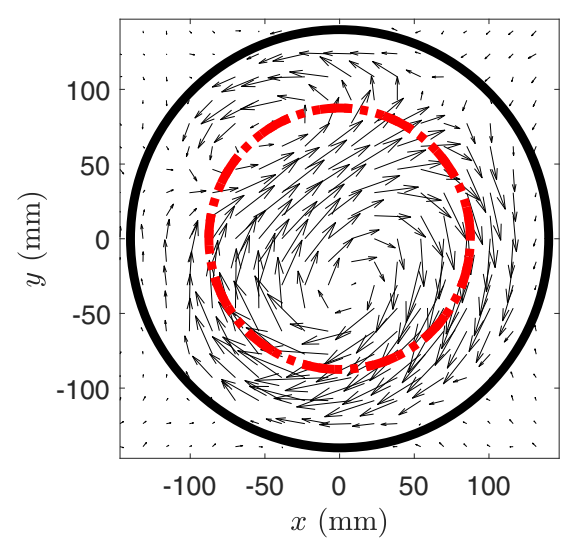

FIG. 11. Criterion used to extract a significant value for the large-scale flow and its direction: the azimuthal velocity is averaged over the ring shown in red. 


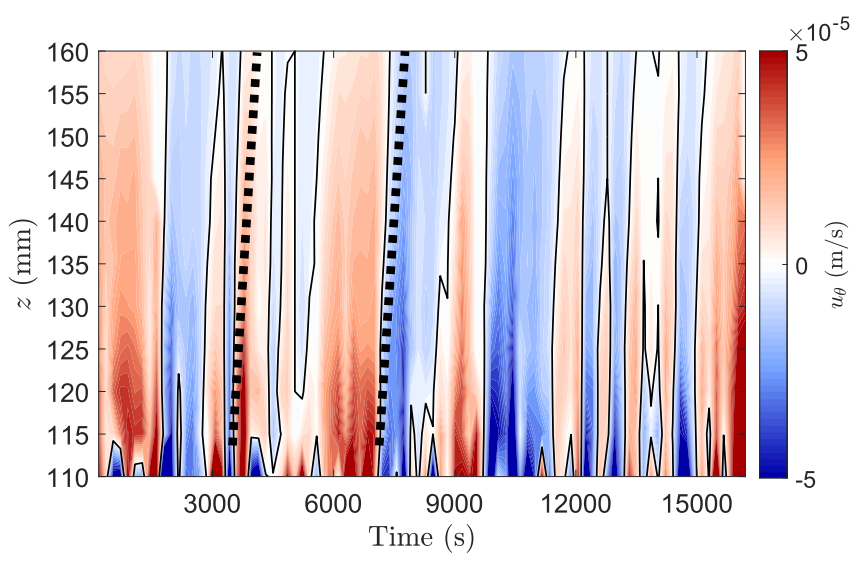

FIG. 12. Reversals of the large-scale flow. $z=115-120 \mathrm{~mm}$ is the convective/buffer layer interface. Ascending plumes often perturb the buffer layer flow. The velocity was measured at 11 heights, marked by each tick in the vertical axis of the figure, and interpolated in between. The slope of the black dot lines represents the viscous coupling phase velocity.

deposits all its energy locally. At $z=z_{c}$, the flow accelerates. Thus the critical height where the flow is intense enough to damp the wave is lowered. As time goes on, this critical height moves towards the location where the waves are emitted. Here, the waves are emitted at the bottom of the stratified layer. We would expect a downward phase propagation if the reversals are driven by IGW nonlinear interactions.

We performed long-time experiments (around 8 hours). Typical results extracted from the criterion described above are shown in Fig. 12. Blue patches (resp. red patches) represent large-scale flow going counterclockwise (resp. clockwise). The present measurements mainly confirm the interpretation of Fig. 9 for the lowest frequencies: the large-scale flow is horizontal and extends over the whole depth of the stratified layer with an amplitude attenuated with height, and exhibits slow reversals. Additionally, some intense events at $z=110 \mathrm{~mm}$ are directly related to penetrative plumes from the convection. Reversal times range from 400 to $1800 \mathrm{~s}$. However, no downward phase propagation of the reversals is observed. On the contrary, the reversals seem to occur along the whole stratification height at the same time, or even with a rapid upward phase propagation. Since the phase propagation is not towards the location where the waves are emitted, the reversals are unlikely to be driven by the nonlinear interactions of IGWs. However, as seen in Sec. III A 3, IGWs propagate in the stratified layer and carry energy. Therefore, they give energy to the large-scale flow through nonlinear interactions. Yet, the process is not dominant in the reversals dynamics.

Since, the reversals observed in Fig. 12 do not have a downward phase propagation, we look for other mechanisms than the QBO mechanism to explain the reversals. Two other mechanisms can be investigated. The first one relies on a specific convective dynamics within the overall stratified layer driven by horizontal gradients related to imperfect top and side boundary conditions. The second mechanism relies on viscous coupling with the underlying convective and buffer layers.

Our fully stratified reference experiment described in Sec. II precludes the first scenario. Indeed, setting the bottom boundary at $10^{\circ} \mathrm{C}$ and the top boundary at $70^{\circ} \mathrm{C}$, no motion is observed for the bottom $3 / 4$ of the tank. In this test experiment, the top $1 / 4$ of the tank is animated by convective motions due to the non homogeneous top heat source (in the standard $4{ }^{\circ} \mathrm{C}$ experiment, where $T_{\text {top }}=$ $35^{\circ} \mathrm{C}$, only $\sim 2 \mathrm{~cm}$ are affected by the convection at the top of the tank, because the nonhomogeneity of the heat source is less important for lower temperature, thus the horizontal convection is weaker). However, these are inefficient to generate waves below and to drive any large-scale flow observable away from the top region. 
This leaves viscous entrainment as a possible driving mechanism. The dotted lines in Fig. 12 show a theoretical viscous time, computed from the time for viscous entrainment to drive $20 \%$ of the horizontal velocity at $z=115 \mathrm{~mm}$ to $z=160 \mathrm{~mm}$, starting from a base state flow at rest. The $20 \%$ value corresponds to the measured value of the large-scale flow at $z=160 \mathrm{~mm}$ compared to the value at $z=115 \mathrm{~mm}$ (noted $u_{b}$ ). The theoretical viscous time entrainment is given by $t=$ $\frac{z^{2}}{4 v \operatorname{erf}^{-2}\left[\left(u / u_{b}-1\right)\right]}$. The reversals occur in a timescale comparable with this theoretical viscous time. The similarity between the slope of the dashed lines and the slope of the upward phases suggests that reversals are driven viscously.

However, the existence of the buffer layer and its associated intense shear, with horizontal velocities opposite to those of the convective layer below (see Fig. 6) precludes direct viscous coupling between the convective and stratified layers. Besides, no reversal has been observed in the convective region. We thus propose a thermal coupling between the convective and buffer layers as seen in Sec. III A 2, associated with a viscous coupling between the buffer and stratified ones. To further quantify this possibility, Figs. 13 and 14 show horizontal velocity fields at different heights and at different times. For each of the columns shown, the first row is the mean flow in the convective layer at depth $z=110 \mathrm{~mm}$, the second row is the mean flow in the buffer layer at depth $z=120 \mathrm{~mm}$, and the third row is the mean flow in the stratified layer at depth $z=145 \mathrm{~mm}$.
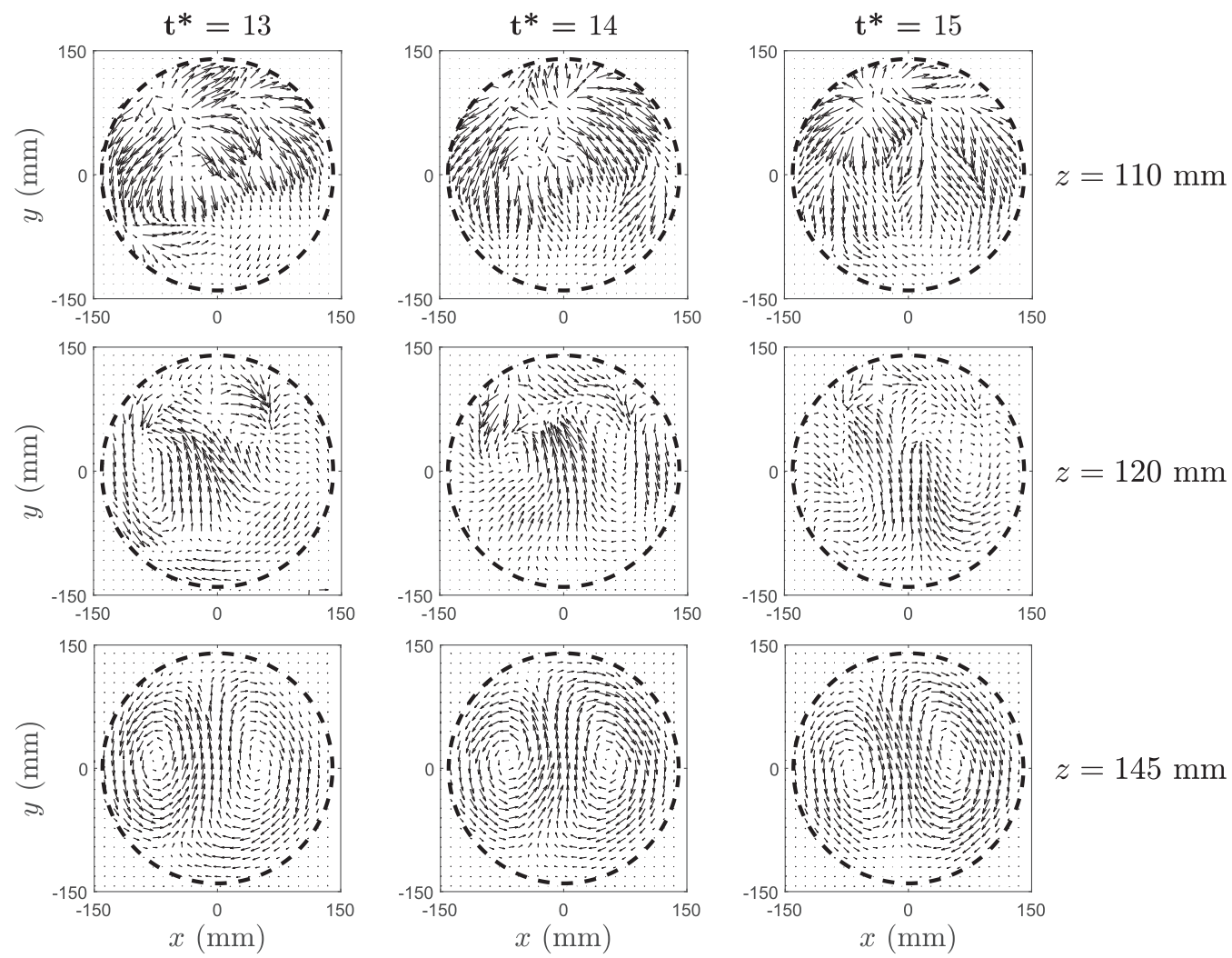

FIG. 13. Velocity vector fields in the horizontal plane. Different columns represent different sweeping cycles $t^{*}$ (one sweeping cycle corresponds to the 11 steps needed to go from the lowest position $z=110 \mathrm{~mm}$ to the highest position $z=160 \mathrm{~mm}$ ). Different rows represent different heights within the same sweeping cycle: the first row is the top of the convection $z=110 \mathrm{~mm}$, the second row is in the buffer layer $z=120 \mathrm{~mm}$, and the third row is in the stratified layer $z=145 \mathrm{~mm}$. Convective plumes are easily noticeable on the first row fields. 

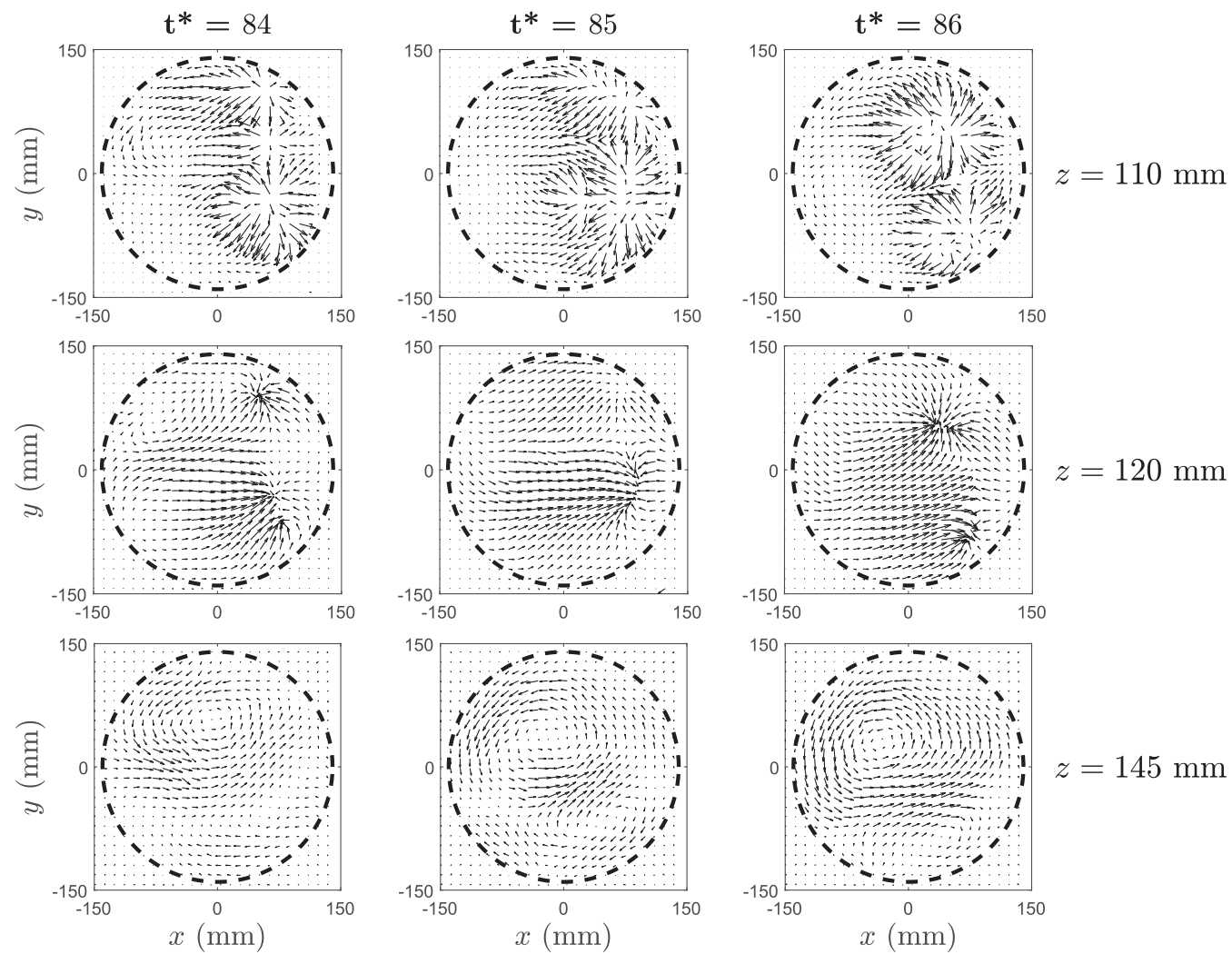

FIG. 14. Same as Fig. 13 but for different sweeping cycles. Note that in this set of velocity fields the buffer and stratified layers are less correlated than they are in Fig. 13.

The correlation coefficients through time between (i) the convective and buffer layers, (ii) the buffer and stratified layers, and (iii) the convective and stratified layers have been computed. They consist of a scalar product of the velocity vector for each position at two different heights rescaled by the product of the norm of the velocity vector at the two heights, i.e.,

$$
R_{i j}=R\left(x_{i}, y_{j}\right)=\frac{u\left(x_{i}, y_{j}, z_{1}\right) \times u\left(x_{i}, y_{j}, z_{2}\right)+v\left(x_{i}, y_{j}, z_{1}\right) \times v\left(x_{i}, y_{j}, z_{2}\right)}{\left[u\left(x_{i}, y_{j}, z_{1}\right)^{2}+v\left(x_{i}, y_{j}, z_{1}\right)^{2}\right]^{1 / 2} \times\left[u\left(x_{i}, y_{j}, z_{2}\right)^{2}+v\left(x_{i}, y_{j}, z_{2}\right)^{2}\right]^{1 / 2}} .
$$

This gives a correlation coefficient $R_{i j}$ for each PIV position in the horizontal plane. The global correlation coefficient $R$ is computed by spatially averaging the local correlation coefficients.

Results are shown in Fig. 15. The convective and buffer layers are negatively correlated: the correlation coefficient is most of the time close to $R=-0.5$. This can also be seen at all times in Figs. 13 and 14, where horizontal velocities in the convective and buffer layers have opposite direction. A diverging flow coming from an impinging plume in the convective zone corresponds to a converging flow in the buffer layer towards the impact zone, hence confirming the thermal coupling mechanism described in Sec. III A 2. This converging flow may lead either to a clockwise or anticlockwise azimuthal mean flow, depending on the details of the chaotic excitation from the convective plumes. The correlation coefficient between the convective and stratified layers can be 


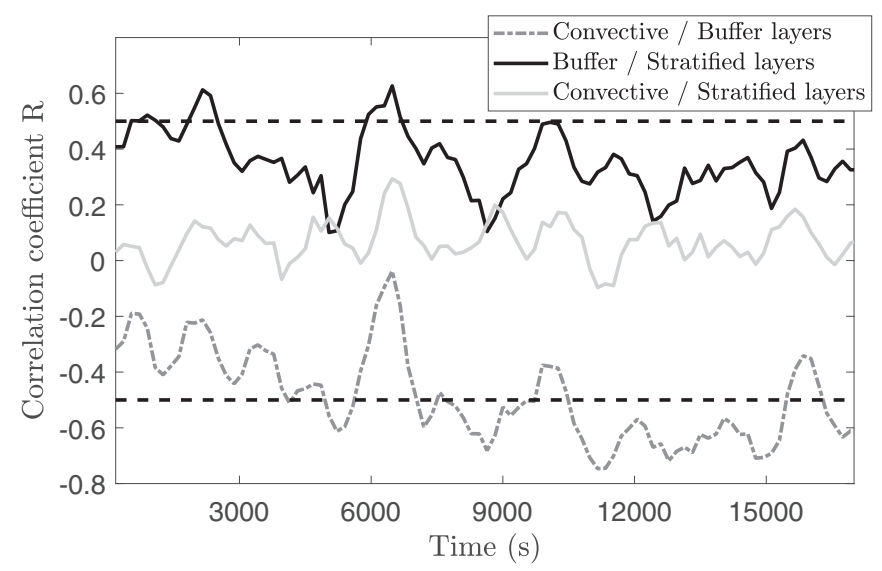

FIG. 15. Velocity correlation between the three layers. Dashed black lines are the -0.5 and 0.5 values. Correlation coefficient are window averaged over 10 min to smooth the curve.

positive or negative, and is anyway most of the time less than 0.2 , in absolute value. The correlation coefficient between the buffer and stratified layers shows a lot of temporal variations. However, it remains always positive. At a given time, the large-scale flow in the stratified layer may switch between a regime strongly dominated by the buffer layer (see also Fig. 13), and a second regime where the flow in the stratified layer is quite different from the flow in the buffer layer (see also Fig. 14).

We thus conclude that the stratified layer is globally viscously driven by the buffer layer. However, the stratified layer exhibits additional complexities. These might be due to IGWs interacting with the large-scale flow. The results from Couston et al. [17] show that the lower the Prandtl number is, the more regular the QBO is. In the experiment, the Prandtl number is close to $\mathrm{Pr}=7$ : the typical associated QBO-type flow is irregular, with low amplitude. We thus propose that large-scale flow driven by IGW nonlinear interaction superimposes on the viscously driven flow, but remains secondary. We do not know at this point how to disentangle those two potential contributions from the available data.

\section{B. Numerical simulations}

The experimental results are not fully sufficient to explain, with complete certainty, the origin of the buffer layer and of the large-scale flow observed in the stratified layer. In addition, the effects of the lateral heat losses and top temperature heterogeneity are difficult to distinguish. To answer these questions, 3D DNS of a configuration similar to our experiments are performed, reproducing the $4{ }^{\circ} \mathrm{C}$ convection but with idealized boundary conditions (i.e., no flux on the sides, and fixed temperature at the top and bottom). As mentioned in Sec. II B, the Rayleigh number Ra and $T_{\text {top }}$ are tuned so that the interface depths in the experiment and the numerical simulation are similar. We have $\mathrm{Ra}=10^{7}$ and $T_{\text {top }}=48^{\circ} \mathrm{C}$. All the numerical simulations are run dimensionless, but results are shown in dimensional values. The length scale is $H=200 \mathrm{~mm}$, the vertical extent of the whole domain (hence diameter is $D=300 \mathrm{~mm}$ ), the timescale is the thermal diffusive time $\tau=\frac{H^{2}}{\kappa}=\frac{0.2^{2}}{1.5 \times 10^{-7}}=2.67 \times 10^{5} \mathrm{~s}$, and the temperature is given by the dimensionless temperature $\theta=\frac{T-T_{i}}{T_{0}-T_{i}}$, where $T, T_{i}, T_{0}$ are respectively the dimensional temperature, the inversion temperature of the equation of state (i.e., $4^{\circ} \mathrm{C}$ ), and the bottom temperature (i.e., $0^{\circ} \mathrm{C}$ ). Results for Secs. III B 1 and III B 2 are computed from a $(x, z)$ vertical plane located along a cylinder diameter. 

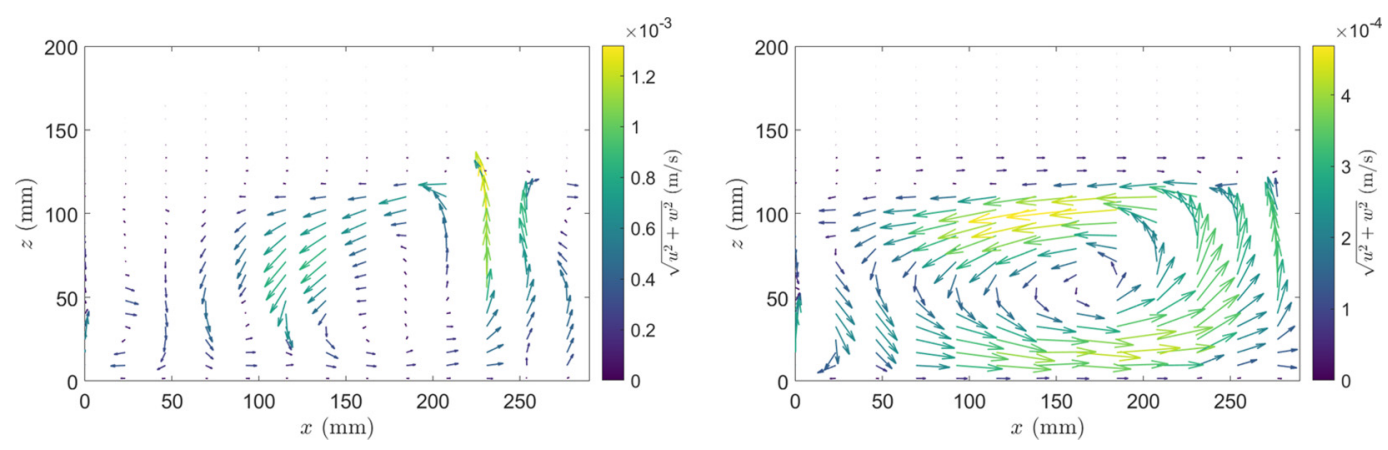

FIG. 16. Left: Instantaneous velocity field. An ascending plume is visible at $x=230 \mathrm{~mm}$. Right: Largescale circulation in the convective layer obtained by time averaging velocities over a 50 minute recording. The large-scale circulation is a counterclockwise cell. Maximum instantaneous velocities are 3 times bigger than the maximum averaged velocities.

\section{Large-scale circulation in the convection zone and buffer layer}

Figure 16 shows that a large-scale circulation takes place in the convective layer. It consists of a cell filling the whole convective layer, and exhibits no reversal over the whole course of the simulation. The fluid rotates counterclockwise in the vertical plane. This is qualitatively consistent with the mean flow observed in the experiment and shown in the right panel of Fig. 2. As in the experiments, a counter current exists on the top of the convective layer at $z=120 \mathrm{~mm}$, creating a strong shear and demonstrating the existence of a buffer layer in the numerical simulation as well.

The space-time diagram of the mean horizontal flow shown in Fig. 17 confirms it. Observing the buffer layer in the absence of side thermal losses and top temperature heterogeneity is an additional argument accounting for the fact that it is not an artifact driven by imperfect experimental conditions. We also observe that the flow within the convection stays positive through time at the bottom and negative at the top. This is evidence of the steady large-scale circulation taking place in the convective layer. Some events appear at $t>1.42 \times 10^{5} \mathrm{~s}$ and are interpreted as quasireversal of the large-scale circulation.
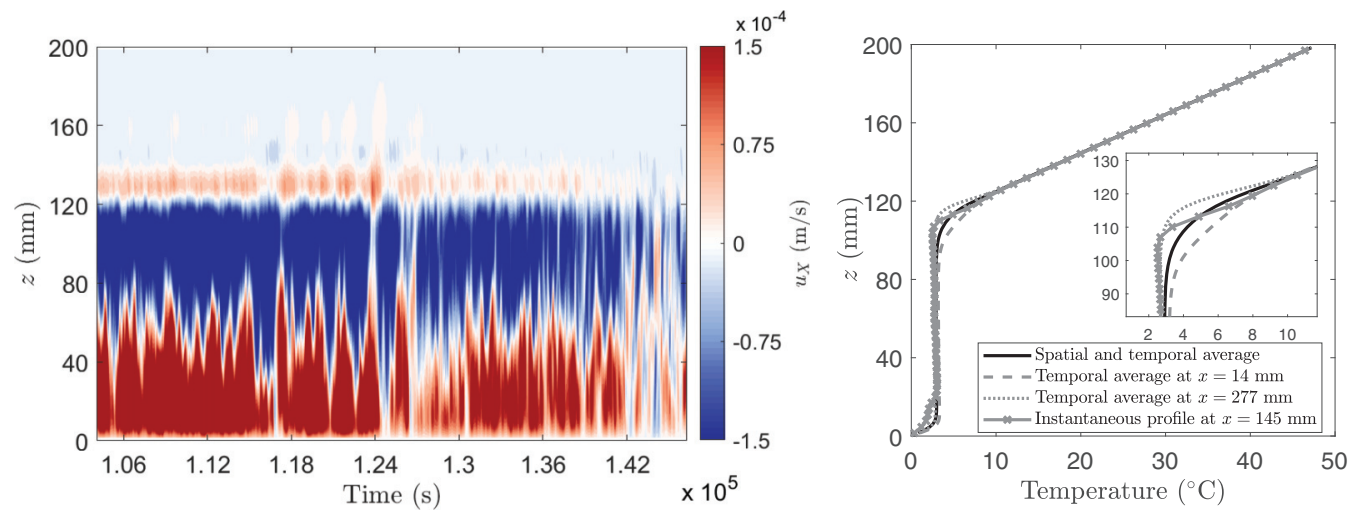

FIG. 17. Left: Horizontal average of the horizontal velocity $u$ over a vertical cross section in the middle of the tank. The buffer layer can be seen above $z=120 \mathrm{~mm}$. A stationary large-scale circulation is present in the convective layer, even if it appears quite perturbed at the end of the signal. Right: Temperature profiles along the $z$ axis. 


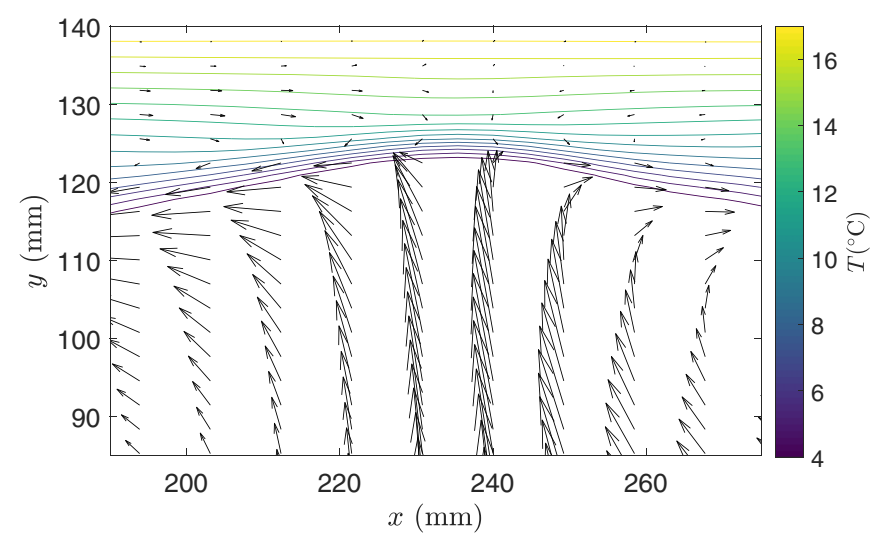

FIG. 18. Velocity field and temperature isotherms at the end of an upward plume impact on the interface.

The temperature profile along the $z$ axis is also plotted on the right panel of Fig. 17. The figure shows a temporal and horizontal average of the temperature field (black thick curve), two temporal averages at two different positions $x=14 \mathrm{~mm}$ (left side of the tank: dashed grey) and $x=277 \mathrm{~mm}$ (right side: dotted grey) and an instantaneous profile at $x=145 \mathrm{~mm}$ (middle of the tank: thick grey with crosses). The thermal boundary layer can be seen, between $z=0 \mathrm{~mm}$ and $z=10 \mathrm{~mm}$. Then, between $z=10 \mathrm{~mm}$ and $z=100 \mathrm{~mm}$ lies a layer of constant temperature $T \sim 2.8^{\circ} \mathrm{C}$. In the range $100 \leqslant z \leqslant 115 \mathrm{~mm}$, the temperature profile evolves from constant to linear for $z>115 \mathrm{~mm}$. The $T=4{ }^{\circ} \mathrm{C}$ (resp. $T=8{ }^{\circ} \mathrm{C}$ ) isotherm is located at $z=110 \mathrm{~mm}$ (resp. $z=120 \mathrm{~mm}$ ). Note that the temporal averages of the temperature profiles are different on the left and right sides of the tank. Indeed, the constant temperature height goes to $z=90 \mathrm{~mm}$ for the left side whereas it goes to $z=115 \mathrm{~mm}$ for the right side. This suggests that the convective/buffer layer interface does not lies at one height over the whole tank but is a function of time and space. This is very likely due to the large-scale circulation. Thus, the thermal coupling described in Sec. III A 2 will likely occur at different heights, depending on time and horizontal position.

The thermal coupling as schematized in Fig. 7 can be found in the numerical simulation. This is represented in Fig. 18. An upward plume impacting the convective/buffer layer interface is seen. The isotherms ranging from $T=4{ }^{\circ} \mathrm{C}$ to $T=11^{\circ} \mathrm{C}$ are deflected upward, due to the plume bringing cold fluid upward. In contrast, the isotherms $T=12-14{ }^{\circ} \mathrm{C}$ are deflected downward by the converging flow. Isotherms at $T \geqslant 15^{\circ} \mathrm{C}$ remain horizontal. After the impact on the interface, the plume is deflected outwards. One could expect the fluid above the impact to be viscously entrained by this outward deflection. However, as observed in Fig. 18 for the simulation and Figs. 13 and 14 for the experiment, the fluid above the interface is going towards the plume, i.e., in the opposite direction of the fluid below, hence explaining the observed shear (see Figs. 18 and 7). The time evolution of these dynamics is shown in Fig. 19.

Figure 19 shows the time evolution of the horizontal velocity $u$ in the shear layer at $z=128 \mathrm{~mm}$ and the time evolution of the vertical velocity $w$ in the convective layer at $z=108 \mathrm{~mm}$. Comparing the two panels of Fig. 19 shows that upward plumes are concomitant with converging horizontal velocities towards the plume impact. Indeed, the spatio-temporal diagram of $w$ exhibits local strong upward plumes. These plumes, as suggested by the dashed black lines, are correlated in time and space with converging horizontal velocities. For instance, an upward plume is seen at $x \approx 220 \mathrm{~mm}$ and $t \approx 1.043 \times 10^{5} \mathrm{~s}$. At the same horizontal position and time, the positive horizontal velocity becomes stronger and the negative horizontal velocity patch increases in size to reach $x \approx 220 \mathrm{~mm}$. The converging horizontal velocities event occurs a short time after the impact of the plumes. Thus, it can be concluded that the plume induces the converging flow, as suggested by our explanation in Sec. III A 2. 


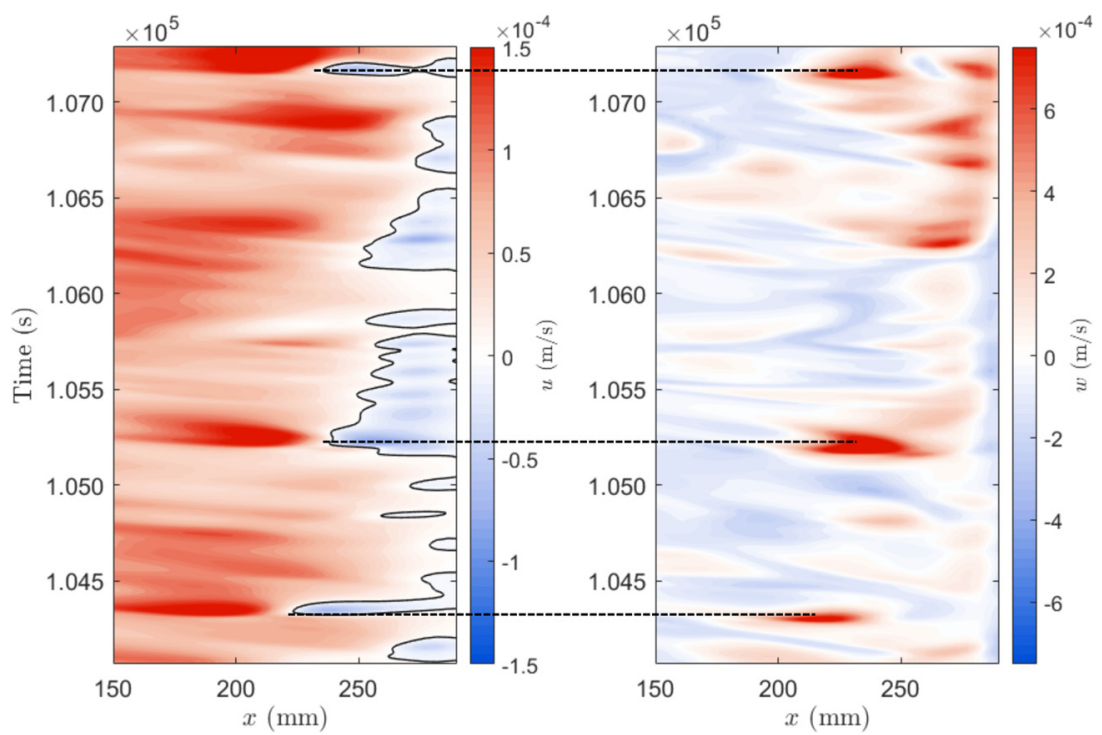

FIG. 19. Left: Spatio-temporal diagram of the horizontal velocity $u$ at $z=128 \mathrm{~mm}$. Right: Spatio-temporal diagram of the vertical velocity $w$ at $z=108 \mathrm{~mm}$. The event at $t \approx 1.052 \times 10^{5} \mathrm{~s}$ is shown in Fig. 18 .

\section{Internal gravity waves}

PSDs are computed in the stratified and buffer layers and are plotted in Fig. 20. As for the experiment (Fig. 9), numerical results show oscillatory motions at different frequencies attenuated with height. Experimental results (Fig. 9) and numerical results (Fig. 20) show strongly similar dynamics: most of the energy is present at low frequencies $\left(f<3 \times 10^{-3} \mathrm{~Hz}\right)$. The motions with frequencies ranging from $3 \times 10^{-3} \mathrm{~Hz}$ to $N$ are less intense, and almost no energy is seen at frequencies $f>N$.

The right panel of Fig. 20 shows two selected vertical profiles (shown by the white dashed line in the left pane) of the PSD rescaled by the PSD at $z=118 \mathrm{~mm}$. The energy for the higher frequency $\left(f=1.4 \times 10^{-2} \mathrm{~Hz}\right)$ decreases slower than the energy for the lower frequency
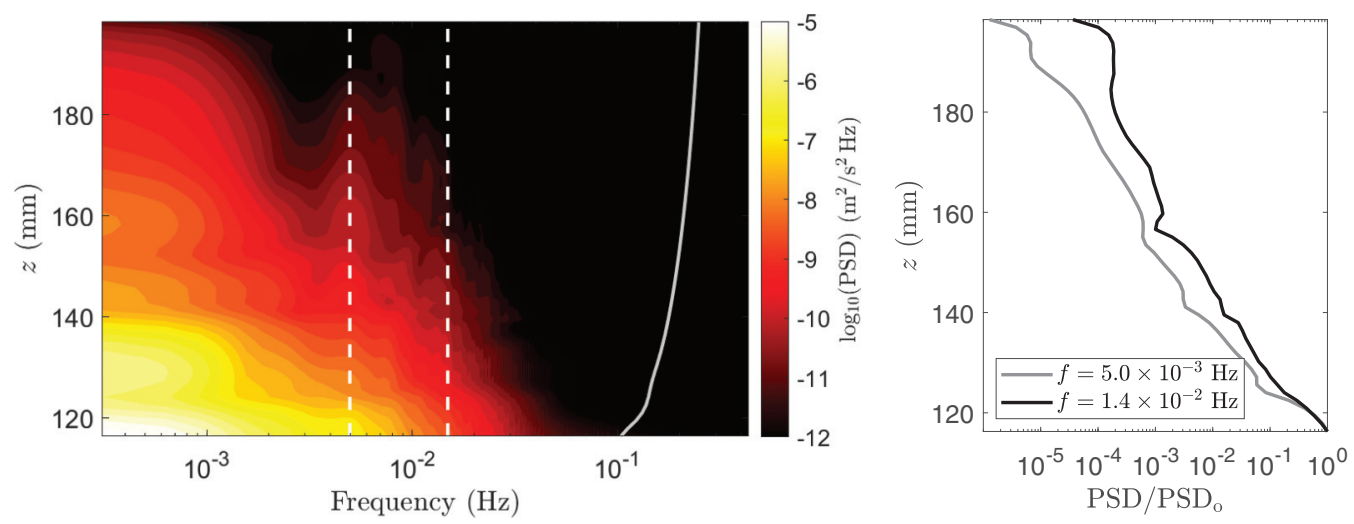

FIG. 20. Left: Power spectral density of the absolute velocity $\sqrt{u^{2}+w^{2}}$ in the buffer and stratified layers. The grey curve shows the buoyancy frequency profile computed from the spatial and temporal average of the temperature field. Right: Two selected profiles (taken at frequencies shown by dashed lines on the left graph) of the rescaled PSDs by the PSD at the top of the convective layer, i.e., $z=118 \mathrm{~mm}$. 

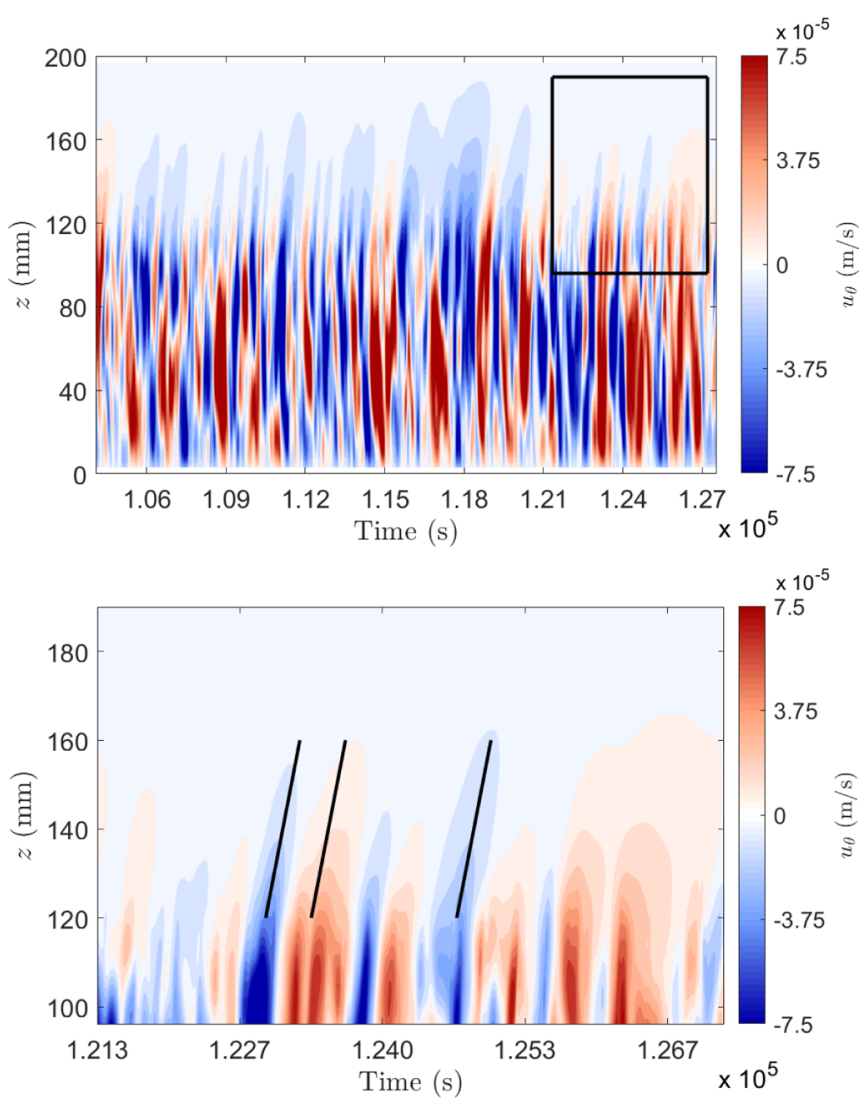

FIG. 21. Spatio-temporal diagrams of the azimuthal averaging of the azimuthal velocity inside a virtual cylinder of radius $r=140 \mathrm{~mm}$. The bottom figure is a zoom on the stratified zone, delimited in the top figure by the black square. The slope of the black lines shows the theoretical viscous diffusive time.

$\left(f=5.0 \times 10^{-3} \mathrm{~Hz}\right)$. This is, like the experimental results, in agreement with the dispersion relation of IGWs. The overall behavior of wave spectra is similar in experiment and numerical simulation, with an attenuation length independent of the frequency in the low-frequency signal thus confirming a viscous coupling origin of the large-scale flow, and increasing when the frequency goes towards $N$ in the wave domain.

\section{Large-scale flow within the stratified layer}

Similarly to what has been done for the experimental data, Fig. 21 shows the mean azimuthal velocity over the whole height of a virtual cylinder of radius $r=140 \mathrm{~mm}$. We observe reversals within the convective layer $(z<120 \mathrm{~mm})$, which are not systematically correlated with the signal in the stratified layer. The mean velocity in the stratified layer also exhibits reversals. They are characterized by an upward phase propagation from the buffer zone at $z=120 \mathrm{~mm}$, as shown in the zoom (bottom panel of Fig. 21). The phase velocity seen in Fig. 21 is in good agreement with the theoretical time for viscous propagation $t=\frac{z^{2}}{4 v \operatorname{erf}^{-2}\left[\left(u / u_{b}-1\right)\right]}$. This corroborates the fact that the reversals observed within the stratified layer are viscously driven from the dynamics occurring in the buffer layer, as has been seen in the experiment. Reversal times range from 300 to $1500 \mathrm{~s}$. Those reversal times are similar to the experimental ones, though slightly shorter (numerical reversals are $\sim 20 \%$ faster than experimental reversals). 


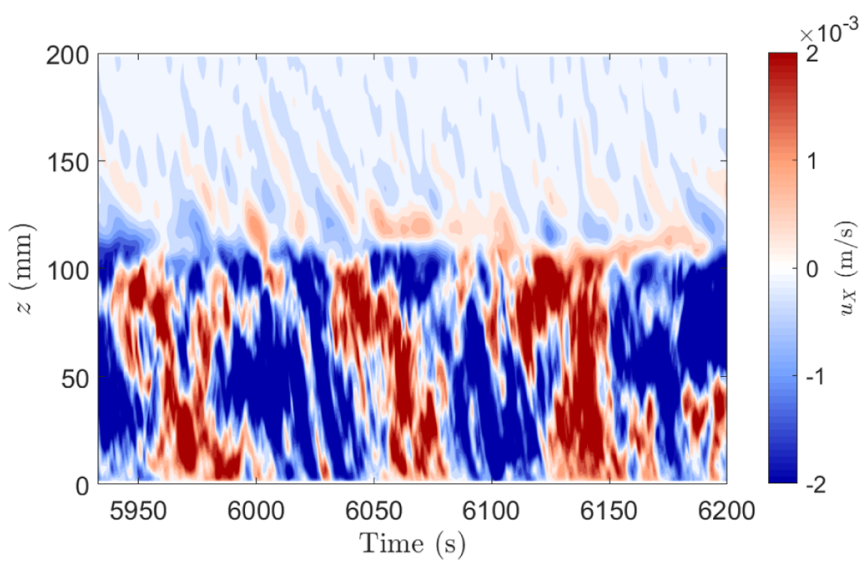

FIG. 22. Horizontal average of the horizontal velocity $u$ over a vertical cross section in the middle of the numerical domain for $\operatorname{Pr}=0.1$.

\section{CONCLUSION}

The $4{ }^{\circ} \mathrm{C}$ convection experiment, originally performed by Townsend [11], has been reinvestigated using long-term PIV measurements in a vertical cross section and in several horizontal cross sections within the stratified layer. This last type of measurement has allowed us to investigate the long-term horizontal mean flow in the stratified layer. Experiments have been complemented by direct numerical simulations. The first result of this paper is the confirmation, in 3D and with ideal boundary conditions, of the presence of a buffer layer, including an overshooting region as first observed by Perrard [13], and a shear region. We have argued that the buffer layer is driven by thermal coupling with the convection, due to the nonlinear equation of state of water, and that this mechanism is a priori related to a Prandtl number larger than 1 . The second result is that the buffer layer viscously drives slow reversals of the horizontal large-scale flow within the stratified layer.

Additionally, IGWs at different frequencies propagate in the stratified layer. They likely interact with the horizontal large-scale flow, and probably also produce a reversing flow, which superimposes on the viscously driven one. From Couston et al. [17], we know that the Prandtl number has a strong influence on this QBO-like mechanism: the lower the Prandtl number, the stronger the amplitude of the QBO. In water, $\operatorname{Pr} \sim 7$, and the expected amplitude of the large-scale QBO flow is weak, hence it is dominated by the viscous driving. Further experimental studies at lower Prandtl number should allow deciphering the two contributions. One could for instance suggest using gas as a working fluid; however, the absence of density reversal around a given temperature will necessitate one to consider either transient experiments like [9,10], or two-gas experiments which might then be prone to double diffusive instabilities. Experimentally, it also remains to understand why the only successful QBO experiment was performed in salty water, hence with a Schmidt number (equivalent to $\mathrm{Pr}$ ) of 700 .

In the meantime, it is straightforward to change the Prandtl number in the numerical simulation of our setup. We have thus run a second simulation with the same Rayleigh number $\mathrm{Ra}=10^{7}$ and top temperature $\theta_{\text {top }}=11$ but with $\operatorname{Pr}=0.1$. In this simulation, as shown in Fig. 22, no buffer layer is observed, but strong signatures of a QBO like mechanism are visible, marked by downward phase propagation of the reversals of the large-scale flow. This configuration thus deserves a more systematic study in the future.

\section{ACKNOWLEDGMENTS}

The authors acknowledge funding by the European Research Council under the European Union's Horizon 2020 research and innovation program through Grant No. 681835-FLUDYCOERC-2015-CoG. Centre de Calcul Intensif d'Aix-Marseille is acknowledged for granting access to 
its high performance computing resources. Computations were also conducted with the support of the HPC resources of GENCI-IDRIS (Grant No. A0060407543).

[1] K. Hirose, S. Labrosse, and J. Hernlund, Composition and state of the core, Annu. Rev. Earth Planet. Sci. 41, 657 (2013).

[2] T. M. Rogers, D. N. C. Lin, and H. H. B. Lau, Internal gravity waves modulate the apparent misalignment of exoplanets around hot stars, Astrophys. J. 758, L6 (2012).

[3] F. P. Bretherton, Momentum transport by gravity waves, Q. J. R. Meteorol. Soc. 95, 213 (1969).

[4] R. A. Plumb, The interaction of two internal waves with the mean flow: Implications for the theory of the quasi-biennial oscillation, J. Atmos. Sci. 34, 1847 (1977).

[5] T. Fouchet, S. Guerlet, D. F. Strobel, A. A. Simon-Miller, B. Bézard, and F. M. Flasar, An equatorial oscillation in Saturn's middle atmosphere, Nature (London) 453, 200 (2008).

[6] C. B. Leovy, A. J. Friedson, and G. S. Orton, The quasiquadrennial oscillation of Jupiter's equatorial stratosphere, Nature (London) 354, 380 (1991).

[7] M. P. Baldwin, L. J. Gray, T. J. Dunkerton, K. Hamilton, P. H. Haynes, W. J. Randel, J. R. Holton, M. J. Alexander, I. Hirota, T. Horinouchi, D. B. A. Jones, J. S. Kinnersley, C. Marquardt, K. Sato, and M. Takahashi, The quasi-biennial oscillation, Rev. Geophys. 39, 179 (2001).

[8] J. K. Ansong and B. R. Sutherland, Internal gravity waves generated by convective plumes, J. Fluid Mech. 648, 405 (2010).

[9] J. W. Deardorff, G. E. Willis, and D. K. Lilly, Laboratory investigation of non-steady penetrative convection, J. Fluid Mech. 35, 7 (1969).

[10] M. Michaelian, T. Maxworthy, and L. Redekopp, The coupling between turbulent, penetrative convection and internal waves, Eur. J. Mech. B 21, 1 (2002).

[11] A. A. Townsend, Natural convection in water over an ice surface, Q. J. R. Meteorol. Soc. 90, 248 (1964).

[12] P. Le Gal, Penetrative convection in water near $4^{\circ} \mathrm{C}$, in Proceedings of IUTAM Symposium On Variable Density Low-Speed Turbulent Flow, edited by L. Fulachier, J. Lumley and F. Anselmet (Kluwer, Dordrecht, 1997), pp. 221-228.

[13] S. Perrard, M. Le Bars, and P. Le Gal, Experimental and numerical investigation of internal gravity waves excited by turbulent penetrative convection in water around its density maximum, in Studying Stellar Rotation and Convection, Lecture Notes in Physics Vol. 865 (Springer-Verlag, Berlin, 2013), p. 239.

[14] M. Le Bars, D. Lecoanet, S. Perrard, A. Ribeiro, L. Rodet, J. M. Aurnou, and P. Le Gal, Experimental study of internal wave generation by convection in water, Fluid Dyn. Res. 47, 045502 (2015).

[15] D. Lecoanet, M. Le Bars, K. J. Burns, G. M. Vasil, B. P. Brown, E. Quataert, and J. S. Oishi, Numerical simulations of internal wave generation by convection in water, Phys. Rev. E 91, 063016 (2015).

[16] L.-A. Couston, D. Lecoanet, B. Favier, and M. Le Bars, Dynamics of mixed convective-stably-stratified fluids, Phys. Rev. Fluids 2, 094804 (2017).

[17] L.-A. Couston, D. Lecoanet, B. Favier, and M. Le Bars, Order Out of Chaos: Slowly-Reversing Mean Flows Emerge from Turbulently-Generated Internal Waves, Phys. Rev. Lett. 120, 244505 (2018).

[18] L.-A. Couston, D. Lecoanet, B. Favier, and M. Le Bars, The energy flux spectrum of internal waves generated by turbulent convection, J. Fluid Mech. 854, R3 (2018).

[19] R. A. Plumb and A. D. McEwan, The instability of a forced standing wave in a viscous stratified fluid: a laboratory analogue of the quasi biennial oscillation, J. Atmos. Sci. 35, 1827 (1978).

[20] B. Semin, G. Facchini, F. Pétrélis, and S. Fauve, Generation of a mean flow by an internal wave, Phys. Fluids 28, 096601 (2016).

[21] B. Semin, N. Garroum, F. Pétrélis, and S. Fauve, Nonlinear Saturation of the Large Scale Flow in a Laboratory Model of the Quasibiennial Oscillation, Phys. Rev. Lett. 121, 134502 (2018).

[22] R. S. Lindzen and J. R. Holton, A theory of the quasi-biennial oscillation, J. Atmos. Sci. 25, 1095 (1968). 
[23] J. R. Holton and R. S. Lindzen, An updated theory for the quasi biennial oscillation cycle of the tropical stratosphere, J. Atmos. Sci. 29, 1079 (1972).

[24] G. Oster, Density gradients, Sci. Am. 213, 70 (1965).

[25] P. Meunier and T. Leweke, Analysis and treatment of errors due to high velocity gradients in particle image velocimetry, Exp. Fluids 35, 408 (2003).

[26] P. Fischer, J. Lottes, and S. Kerkemeier, Nek5000 v17.0, 2017, https://nek5000.mcs.anl.gov.

[27] L. N. Howard, Convection at high Rayleigh number, Applied Mechanics, edited by H. Görtler (Springer, Berlin, 1966), pp. 1109-1115. 